\title{
Optimization in liner shipping
}

Brouer, Berit Dangaard; Karsten, Christian Vad; Pisinger, David

Published in:

4 O R

Link to article, DOI:

$10.1007 / \mathrm{s} 10288-017-0342-6$

Publication date:

2017

Document Version

Peer reviewed version

Link back to DTU Orbit

Citation (APA):

Brouer, B. D., Karsten, C. V., \& Pisinger, D. (2017). Optimization in liner shipping. 4 O R, 15(1), 1-35. https://doi.org/10.1007/s10288-017-0342-6

\section{General rights}

Copyright and moral rights for the publications made accessible in the public portal are retained by the authors and/or other copyright owners and it is a condition of accessing publications that users recognise and abide by the legal requirements associated with these rights.

- Users may download and print one copy of any publication from the public portal for the purpose of private study or research.

- You may not further distribute the material or use it for any profit-making activity or commercial gain

- You may freely distribute the URL identifying the publication in the public portal

If you believe that this document breaches copyright please contact us providing details, and we will remove access to the work immediately and investigate your claim 


\title{
Optimization in liner shipping
}

Berit Dangaard Brouer, Christian Vad Karsten, and David Pisinger

\begin{abstract}
Seaborne trade is the lynchpin in almost every international supply chain, and about $90 \%$ of non-bulk cargo worldwide is transported by container. In this survey we give an overview of data-driven optimization problems in liner shipping. Research in liner shipping is motivated by a need for handling still more complex decision problems, based on big data sets and going across several organizational entities. Moreover, liner shipping optimization problems are pushing the limits of optimization methods, creating a new breeding ground for advanced modelling and solution methods. Starting from liner shipping network design, we consider the problem of container routing and speed optimization. Next, we consider empty container repositioning and stowage planning as well as disruption management. In addition, the problem of bunker purchasing is considered in depth. In each section we give a clear problem description, bring an overview of the existing literature, and go in depth with a specific model that somehow is essential for the problem. We conclude the survey by giving an introduction to the public benchmark instances LINER-LIB. Finally, we discuss future challenges and give directions for further research.
\end{abstract}

Berit Dangaard Brouer

Optivation, Rued Langgaards Vej 7, 2300 Copenhagen S, Denmark e-mail: berit.dangaard.brouer@optivation.dk

Christian Vad Karsten

DTU Management Engineering, Technical University of Denmark, Produktionstorvet 424, DK-2800 Kgs. Lyngby, Denmark e-mail: chrkr@dtu.dk

David Pisinger

DTU Management Engineering, Technical University of Denmark, Produktionstorvet 424, DK-2800 Kgs. Lyngby, Denmark e-mail: dapi@dtu.dk 


\section{Introduction}

Seaborne trade constitutes nearly $80 \%$ of the world trade and provides one of the most energy efficient modes of transportation. As seen in Figure 1, container vessels are up to six times more energy efficient than trains, 15 times more efficient than trucks, and almost 200 times more efficient than planes. A prerequisite to achieve this energy efficiency is to be able to fully utilize the available capacity of the large container vessels. Exploiting the capacity in an efficient manner means lower transportation costs, which will benefit the entire supply chain from producers to consumers. The economical incentive to provide this solution lies with the carrier. However, the huge size of seaborne trade and the complexity of the transportation network makes it necessary to have effective decision support tools at all levels of the operation to achieve an efficient transportation network considering operational cost, fuel efficiency and a short transportation time.
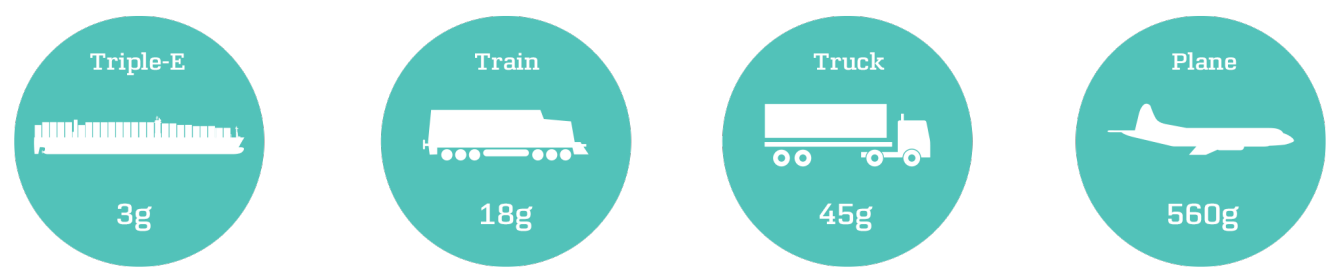

Fig. 1 Grams of $\mathrm{CO} 2$ emitted by transporting 1 tonne of cargo $1 \mathrm{~km}$, using respectively container vessels, trains, trucks or planes. (Source: Maersk, 2015)

Modern container vessels can handle up to 20,000 Twenty-foot Equivalent Units (TEU). Leading companies operate a fleet of more than 500 vessels and transport more than 10,000,000 full containers annually. The huge pressure to fill capacity and utilize the efficiency benefits of the larger vessels is challenged by volatile markets for cargo transport and fierce competition leading to ever changing conditions. Dynamic and flexible operation of a liner shipping network requires advanced decision support tools based on OR techniques. OR techniques to optimize transportation networks have successfully been implemented across the transportation industry, and the size of the maritime transportation network requires techniques that are flexible and scalable. Large scale maritime optimization problems are found both within transportation of bulk cargo, liquefied gases and particularly within liner shipping due to the vast size of the network that global carriers operate. This survey gives an overview of a broad collection of planning problems faced by a global carrier providing a complex transportation network for containerized goods. The solution techniques for the planning problems surveyed exploit recent advances in large scale optimization methods utilizing special problem structures such as separable/independent subproblems and give examples of advanced heuristics using divide-and-conquer paradigms, decomposition and mathematical programming.

Maritime logistics companies encounter large scale planning problems at both the strategic, tactical, and operational level. In liner shipping the boundaries between strategic and tactical, and tactical and operational are not always clear, but Figure 2 shows a rough classification of some of the main planning problems. Among the most important strategic decisions are the decisions of which markets to serve, the fleet size, the composition of the fleet (mix), and the actual design of the sailing routes (network design). The tactical problems include service selection and cargo routing, as well as as fleet deployment, speed optimization, and scheduling of the fleet. Key operational decisions subsume berthing of vessels in ports, stowage of containers on the vessels, as well as empty container repositioning and disruption management. These decisions are important for a network to be operated in the best possible way. In the OR literature the problems are often treated somewhat independently (Christiansen et al., 2013; Meng et al., 2014), but as will be seen throughout this survey the problems are often highly dependent. E.g. the best design of a set of 
services depends very much on the sailing speed on each leg, which again influences the level of service a customer receives through the optimal cargo routing and vice versa. However, solving each of the problems independently is challenging enough and valuable insights can be obtained by considering each problem, or a subset of these, independently. The planning problems can also be classified according to different operational conditions (Kjeldsen, 2011). For a comprehensive review of the OR literature focusing on liner shipping planning problems and a characterization of these, we refer to Meng et al. (2014).

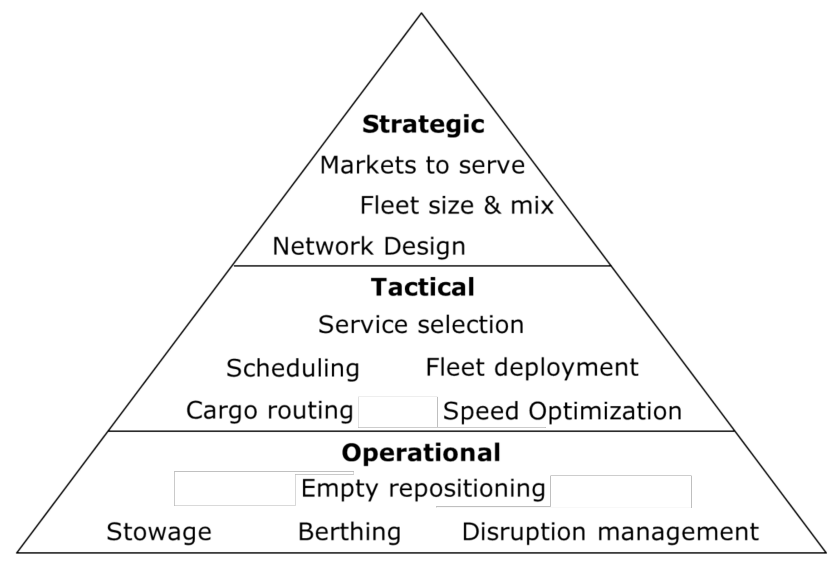

Fig. 2 Main planning problems in liner shipping, grouped in strategic, tactical and operational problems.

Most data in liner shipping are associated with some degree of uncertainty. First of all, demands are fluctuating over the year, and in liner shipping no fees are paid if the customer does not deliver the booked number of containers, so customers may at any time choose to use another shipping company, or to postpone the delivery. As a result, carriers expect significant downfall in the number of ordered containers and this stimulates overbooking from the carriers side. Both contribute to significant uncertainty in the data used as input for the mathematical models. Liner shipping networks also suffer badly from disruptions due to weather and complex technical port operations. As a result, port availabilities are also highly uncertain. If a vessel sticks to the normal time table, it can generally be assumed that the time slot is available, but if a vessel is delayed or the company wants to change the route, all port calls must be negotiated with the port authorities. This substantially complicates planning, requiring flexible planning and the ability to find good alternatives to an existing plan in a time efficient manner.

There are several different approaches for solving large scale optimization problems in liner shipping. If a problem exhibits a special separable structure it can be decomposed and solved more efficiently by using either column generation, if the complication involves the number of variables, or row generation, if the number of constraints is too large (Barnhart et al., 1998; Costa, 2005; Desrosiers and Lübbecke, 2005), or constraint programming (Rossi et al., 2006). For less structured or extremely large problems it can be advantageous to use (meta)heuristics to obtain solutions quickly, but often of unknown quality (Burke and Kendall, 2014; Gendreau and Potvin, 2010). Finally it is frequently possible, with a good modeling of a problem, to rely solely on Linear Programming (LP), or Mixed Integer Programming (MIP), solvers. See e.g. Vielma (2015) for a discussion of modeling techniques and the trade-off between stronger versus smaller models. Algorithmic and hardware improvements have over the last three decades resulted in an estimated speed-up for commercial MIP solvers of a 200 billion factor (Bixby, 2012), making it feasible not only to solve large linear models, but also more advanced integer decision models of realistic size. In practice a combination of the different techniques is often seen and maritime logistics gives an illustrative case of the importance of all of these large scale optimization methods. 
This survey is organized as follows: In section 2 we address the Liner Shipping Network Design Problem. Constructing an efficient network for transporting containers from their source to destination is the backbone of liner shipping. We give an overview of solution methods, and go in depth with some of the most successful algorithms for network design and container routing. Section 3 deals with speed optimization in liner shipping. Since fuel consumption grows cubically with the speed, it is important to find appropriate speeds for each leg while ensuring fast delivery to the customers. Section 4 considers the empty container repositioning problem. Due to the imbalance between trade zones, empty containers tend to pile up at various locations, while being in short supply at other locations. A planning tool for repositioning empty containers using the excess capacity on vessels is needed to provide empty containers at ports with demand. In Section 5, we consider the problem of stowing containers on a vessel and present an optimization model that finds a correct allocation of the containers. Section 6 considers the problem of when and where vessels should purchase their fuel (bunker). Due to price differences between ports, and various contracts with fuel companies, substantial savings can be achieved by proper planning. In Section 7, we study decision support tools for disruption management. As about $70-80 \%$ of all vessels encounter a delay during a round trip, it is essential to develop decision support tools for handling disruptions efficiently. Finally, in Section 8 we introduce the LINER-LIB benchmark sets. Although the data set is designed for liner shipping network design, they can also be used as basis for many of the other problems described here. Section 9 concludes the survey and discuss future challenges. This survey is partially based on the book chapter Brouer et al. (2016).

\section{Liner shipping network design}

Container vessels usually operate along established routes following regular time tables published several months ahead. The routes may not change significantly for years in terms of the markets they serve, but the current sequence of port calls, the frequency of service as well as the time table might be more dynamic in nature. Therefore, the design of the routes is one of the most important strategic decisions. In practice, routes are often operated in cooperation with other companies, which adds an additional dimension of complexity to the design process. The problem of constructing routes and choosing which routes to serve is usually referred to as the Liner Shipping Network Design Problem (LSNDP). The core problem associated with liner shipping network design is to determine which ports the container vessels should visit and in which order. Furthermore, the frequency of the routes must be determined along with the size and speed of the vessels used. A route with a given time table is denoted a service. It is common to have weekly frequency of the services, as this decreases the complexity of coordinating with terminals and connecting services. Of course some services have a different frequency, e.g., bi-weekly. As most services have round trip times exceeding the frequency of service, similar vessels are often grouped to sail the same service at the chosen frequency. The transformation from a generic network of interconnecting rotations into a physical sailing network is mainly concerned with an actual scheduling of the services, deploying vessels from the available fleet and deciding on the speed and actual flow of containers on services. These tactical/operational decisions can be seen as an integrated part of the network design process. Integration would potentially result in higher quality networks, but it is currently not computationally tractable to integrate these decisions. In the following, we describe the LSNDP in a general version, but assuming that the services are operated at a weekly frequency.

Given an estimate of the demands to be transported and a set of possible ports to serve, a carrier wants to design routes for a given fleet of vessels and select which demands of containers to satisfy. A service consists of a set of similarly sized vessels sailing on a non-simple cyclic itinerary of ports according to a fixed weekly schedule. Hence the round trip duration for a vessel is assumed to be a multiple of a week, and to ensure weekly frequency in the serviced ports, a sufficient number of vessels is assigned. To make 
schedules more robust, buffer time is included to account for delays. An example of a service can be seen in Figure 3 which shows the Asia-Europe Service with a round trip time of 12 weeks. The weekly departures may in some cases simplify the mathematical formulation of the problem, since customer demands and vessel capacities follow a weekly cycle. Trunk services serve central main ports, and can be both inter and intra regional, whereas feeder services serve a distinct market and typically visit one single main port and several smaller ports. When the network has been determined the containers can be routed according to a fixed schedule with a predetermined trip duration. A given demand is loaded on to a service at its departure port, which may bring the demand directly to the destination port or the container can be unloaded at one or several intermediate ports for transshipment to another service before reaching its final destination. Therefore, the design of the set of services is complex, as they interact through transshipments, and the majority of containers are transshipped at least once during transport. A carrier aims for a network with high utilization, a low number of transshipments, and competitive transit times. Services are divided into a head- and a backhaul direction due to the asymmetry of world trade. The headhaul direction is the most cargo intensive and vessels are almost full. Hence, the headhaul generates the majority of the revenue and due to customer demand for fast delivery the headhaul operates at increased speeds with nearly no buffer time for delays. The backhaul operates at slower speeds with additional buffer time assigned. A delay incurred on the headhaul is often recovered during the back-haul.

In practice, a carrier will never re-design a network from scratch as there are significant costs associated with the reconfiguration (Tierney et al., 2013). Rather, the planners or network design algorithms will take the existing network and suggest incremental changes to adjust the network to the current economic environment. Most network changes require evaluation of the full cargo routing problem to evaluate the quality of the network since regional changes can have unintended consequences in the entire network.

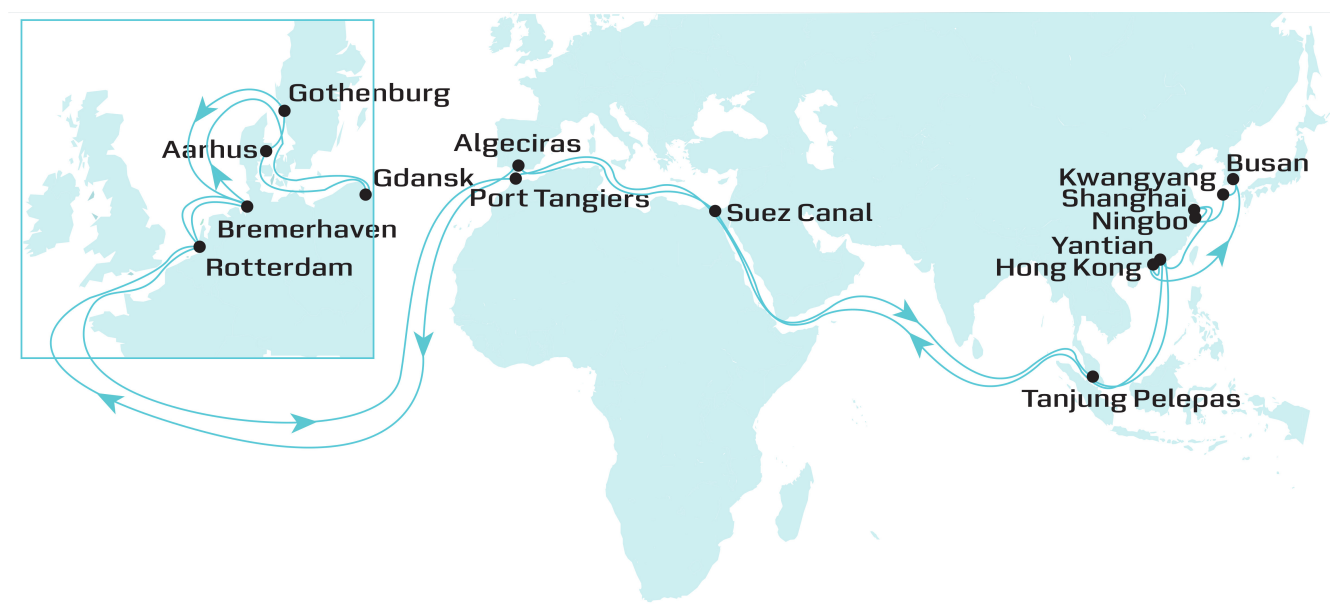

Fig. 3 The Asia-Europe Service (AE10) from the 2016 Maersk Line Network. The round trip takes 12 weeks; hence 12 vessels are deployed to the service to ensure weekly departures. Picture: Maersk Line.

The most general version of the problem does not impose a specific network structure such as huband-spoke, and allows for container transshipment operations between connecting services. Agarwal and Ergun (2008) present one of the first scalable approaches to this problem. Furthermore, they are the first to include transshipments. The model is formulated for a time-space graph cycling over a time span of one week and creates routings for a set of vessel classes. It is one of the only network design models to also include a rough schedule. However, the formulation does not model transshipment costs nor does it include travel time considerations in the container routing although a time-space graph is used. Alvarez (2009) formulates a MIP model including transshipment costs, but the model cannot correctly account 
for transshipment costs on more complex routes, e.g. butterflies as pointed out by Brouer et al. (2014a). The model reduces to a linear multi-commodity flow problem when the integer variables related to vessel rotations are fixed. A tabu search is devised to move between different integer solutions where routes are generated by column generation and the flow problem is solved by an interior point method. The solution of the multi-commodity flow problem is identified as a general bottleneck in local search methods as excessive time is spent on the solution of this. Brouer et al. (2014a) extend the work of Alvarez (2009) and propose a model where transshipment costs are correctly accounted for. Like Alvarez (2009) the problem is solved using tabu search, which only explores a limited part of the solution space for instances of realistic size. Brouer et al. (2014b) propose a new solution method for the model proposed by Brouer et al. (2014a). Based on an initial set of routes an improvement (mat)heuristic based on the solution of a MIP model is proposed. To date the most successful approaches have been heuristic, but some attempts have been made at creating exact methods for the problem. Reinhardt and Pisinger (2012) proposed the first branch-and-cut approach to the problem that considers transshipment costs. The method is able to create simple as well as non-simple butterfly routes. Results are reported for instances with up to 15 ports.

Early work on network design (Rana and Vickson, 1991; Fagerholt, 1999) did not consider transshipments because their importance used to be much less significant from an industry perspective. The model by Rana and Vickson (1991) was later extended by Shintani et al. (2007). The extension relaxed a restrictive visiting order to represent a more realistic set of routes and repositioning of empty containers is also included, but transshipments are still not considered. To solve the problem a genetic algorithm is used. Later, the importance of transshipments in modern liner shipping networks has been emphasized (Baird, 2006; Notteboom and Rodrigue, 2008; Brouer et al., 2014a). Other contributions consider less general versions of the problem, e.g. in a version with only a single route or a version with a set of routes, but no transshipments allowed. Other approaches identify some ports as hub ports and feeder ports are assigned hubs where they can transship a priori. For a review on the literature on the more restricted problems we refer to Christiansen et al. (2013).

Gelareh et al. (2010) have a more game theoretical approach to network design and investigate the competitive position of a carrier in a market where a newcomer carrier is entering. They propose a network design model with a hub-and-spoke structure, where the market share is determined by cost and transit time. It is stressed that it is important to consider both transit time and cost in the construction of a liner shipping network.

Routing of both vessels and containers are in most state-of-the-art methods considered simultaneously (Agarwal and Ergun, 2008; Alvarez, 2009; Brouer et al., 2014b; Reinhardt and Pisinger, 2012; Shintani et al., 2007), as these problems are completely interrelated. However, several of the before mentioned approaches exploit the fact that the problems are separable into two tiers, and design algorithms utilizing this structure. The cargo routing reduces to a multi-commodity flow problem, MCF, and serves as the lower tier where the revenue of the network is determined. The vessel routing problem reduces to a (more complex) problem of cycle generation and corresponds to the upper tier, where the cost of the network is determined. The following section gives insight to the container routing problem, and its relation to the multi-commodity flow problem.

\subsection{Container routing}

At the heart of many of the planning problems in liner shipping lies the problem of cargo routing. Mathematically, the problem can be formulated as an extension of the multi-commodity flow problem.

We define $G=(N, A)$ to be a directed graph with nodes $N$ and edges $A$. The node set $N$ represents the geographical locations in the model, i.e., ports and the arc set $A$ connects the ports. The arcs are determined by the scheduled itineraries and the cargo capacity is determined by the assignment of vessels to the sched- 
ule. Let $K$ be the set of commodities to transport, $q^{k}$ be the amount of commodity $k \in K$ that is available for transport, and $u_{i j}$ be the capacity of edge $(i, j)$. We assume that each commodity has a single origin node, $O_{k}$, and a single destination node, $D_{k}$.

There are two commonly used formulations of the MCF based on either arc or path flow variables. The arc flow formulation can be stated as follows. For each node $i \in N$ and commodity $k \in K$ we define $q(i, k)=q^{k}$ if $i=O_{k}, q(i, k)=-q^{k}$ if $i=D_{k}$, and $q(i, k)=0$ otherwise. For each node $i \in N$ we define the set of edges with tail in node $i$ as $\delta^{+}(i)=\left\{\left(j, j^{\prime}\right) \in A: j=i\right\}$ and head in node $i$ as $\delta^{-}(i)=\left\{\left(j, j^{\prime}\right) \in A: j^{\prime}=i\right\}$. The cost of traversing an $\operatorname{arc}(i, j) \in A$ is $c_{i j}^{k}$ and the variables of the model, $x_{i j}^{k} \geq 0$ expresses the flow of commodity $k$ on arc $(i, j) \in A$.

With this notation the MCF problem can be stated as the following LP

$$
\begin{array}{lll}
\min & \sum_{(i, j) \in A} \sum_{k \in K} c_{i j}^{k} x_{i j}^{k} & \\
\text { s.t. } & \sum_{\left(j, j^{\prime}\right) \in \delta^{+}(i)} x_{j j^{\prime}}^{k}-\sum_{\left(j, j^{\prime}\right) \in \delta^{-}(i)} x_{j j^{\prime}}^{k}=q(i, k) & i \in N, k \in K \\
& \sum_{k \in K} x_{i j}^{k} \leq u_{i j} & (i, j) \in A \\
& x_{i j}^{k} \geq 0 & (i, j) \in A, k \in K
\end{array}
$$

The objective function (1) minimizes the cost of the flow. The flow conservation constraint (2) ensures that a commodity flows from its origin to its destination node. The capacity constraint (3) ensures that the capacity of each edge is respected. The formulation has $|K||A|$ variables and $|A|+|K||N|$ constraints. The number of variables is hence polynomially bounded, but for large graphs like the ones seen in global liner shipping networks this formulation requires excessive computation time and may even be too large for standard LP-solvers (see e.g. Brouer et al. (2011)).

The block-angular structure of the constraint matrix in the arc-flow formulation can be exploited and by Dantzig-Wolfe decomposition it is possible to get a reformulation with a master problem considering paths for all commodities, and a subproblem defining the possible paths for each commodity $k \in K$. We note that in general any arc flow can be obtained as a convex combination of path flows. In the path-flow formulation each variable, $f^{p} \geq 0$, in the model corresponds to a path, $p$, through the graph for a specific commodity. The variable states how many units of a specific commodity are routed through the given path. The cost of each variable is given by the parameter $c_{p}$. Let $P^{k}$ be the set of all feasible paths for commodity $k, P^{k}(a)$ be the set of paths for commodity $k$ that use edge $a$, and $P(a)=\cup_{k \in K} P^{k}(a)$ be the set of all paths that use edge $a$. The model then becomes:

$$
\begin{aligned}
& \min \sum_{k \in K} \sum_{p \in P^{k}} c_{p} f^{p} \\
& \text { s.t. } \sum_{p \in P^{k}} f^{p}=q_{k} \quad k \in K \\
& \sum_{p \in P(a)} f^{p} \leq u_{i j} \quad a=(i, j) \in A \\
& f^{p} \geq 0 \quad k \in K, p \in P^{k}
\end{aligned}
$$

The objective function (5) again minimizes the cost of the flow. Constraint (6) ensures that the demand of each commodity is met and constraint (7) ensures that the capacity limit of each edge is obeyed. The path-flow model has $|A|+|K|$ constraints, but the number of variables is, in general, exponentially growing with the size of the graph. However, using column generation the necessary variables can be generated dynamically and in practice the path-flow model can often be solved faster than the arc-flow model for large scale instances of the LSNDP problem (Brouer et al., 2011). 
Column generation operates with a reduced version of the LP (5)-(8), which is called the master problem. The master problem is defined by a reduced set of columns $Q^{k} \subseteq P^{k}$ for each commodity $k$ such that a feasible solution to the LP (5)-(8) can be found using variables from $\cup_{k \in K} Q^{k}$. Solving this LP gives rise to dual variables $\pi_{k}$ and $\lambda_{i j}$ corresponding to constraint (6) and (7), respectively.

For a path $p \in \cup_{k \in K} P^{k}$, let $\kappa(p)$ denote the commodity that the path serves. Then we can calculate the reduced cost $\bar{c}_{p}$ of each path $p \in \cup_{k \in K} P^{k}$ as follows

$$
\bar{c}_{p}=\sum_{(i, j) \in p}\left(c_{i j}^{\kappa(p)}-\lambda_{i j}\right)-\pi_{\kappa(p)} .
$$

If we can find a path $p \in \cup_{k \in K}\left(P^{k} \backslash Q^{k}\right)$ such that $\bar{c}_{p}<0$ then this path has the potential to improve the current LP solution and should be added to the master problem, which is resolved to give new dual values. If, on the other hand, we have that $\bar{c}_{p} \geq 0$ for all $p \in \cup_{k \in K}\left(P^{k} \backslash Q^{k}\right)$ then we know the master problem defined by $Q^{k}$ provides the optimal solution to the complete problem (for more details see Karsten et al. (2015c)).

In order to find a variable with negative reduced cost or prove that no such variable exists, we solve a sub-problem for each commodity. The sub-problem seeks the feasible path for commodity $k$ with minimum reduced cost given the current dual values. Solving this problem amounts to solving a shortest path problem from source to destination of the commodity with edge costs given by $c_{i j}-\lambda_{i j}$ and subtracting $\pi_{k}$ from this cost in order to get the reduced cost. As will be seen later, we can extend the model to reject demands by including additional variables with an appropriate penalty. When solving the shortest path problem additional industry constraints such as number of transshipments, trade policies, or time limits on cargo trip duration can be included. Including such constraints will increase the complexity of the sub-problem as the resulting problem becomes a resource constrained shortest path problem. Karsten et al. (2015c) proposed a tailored algorithm for a cargo routing problem considering lead times and showed that it does not necessarily increase the solution time to include transit time constraints, mainly because the size of the solution space is reduced. Additionally, Karsten et al. (2015c) give an overview of graph topologies accounting for transshipment operations when considering transit times.

In the next section we describe a more recent approach to construct routes used in the upper tier of the network design problem, which uses an advanced mathematical programming based heuristic to solve the problem within a large scale search framework.

\subsection{Matheuristic for liner shipping network design}

The LSNDP problem involves routing of vessels and flowing of containers, where we have seen that flowing of containers can be done quite efficiently. Algorithms for LSNDP can therefore roughly be divided into four categories depending on the coupling between vessel routing and flowing of containers.

- Integrated approaches design routes and flow containers at the same time. As a consequence optimal methods can only solve very insignificant instances of the LSNDP (Alvarez, 2009; Reinhardt and Pisinger, 2012) or provide lower bounds (Plum et al., 2014).

- Route-first-flow-next approaches apply a two-step approach where routes are generated in the first step, and containers are flown in the second step. These algorithms include (Agarwal and Ergun, 2008; Alvarez, 2009; Brouer et al., 2014b; Karsten et al., 2015a, 2016).

- Flow-first-route-next approaches also apply the two-step approach, but relax the route constraints so that cargo can be flown through the network on independent edges. Based on the calculated network flow, routes can be constructed that cover the demand (Pisinger, 2016). 
- Merging of routes. This approach assumes that a set of good candidate routes are provided by a manual planner or by some constructive algorithms. The model is then able to construct an overall LSNDP minimizing the operational costs (Balakrishnan and Karsten, 2015a,b).

Currently, approaches based on the route-first-flow-next decomposition are able to solve the largest problems.

Several algorithms for solving larger instances of the LSNDP can be categorized as matheuristics combining mathematical programming with metaheuristics exploiting the two tier structure, where the variables of the upper tier describe a service while the variables of the lower tier describe the container routing (for a reference model of the LSNDP see Brouer et al. (2014a)). Agarwal and Ergun (2008) apply a heuristic Benders' decomposition algorithm as well as a Branch and Bound algorithm on a reasonable subset of feasible routing variables, Alvarez (2009) applies a tabu search scheme, where the routing variables are generated by a mathematical program based on the dual values of the lower tier MCF problem in each iteration. Brouer et al. (2014a) use a heuristic column generation scheme, where the routing columns are generated by an integer program based on information from both tiers of the LSNDP along with a set of business rules. The integer program in (Brouer et al., 2014a) constructs a single, possibly non-simple, cyclic route for a given service configuration of vessel class and speed. Route construction is based on the Miller-Tucker-Zemlin subtour elimination constraints known from the CVRP to enumerate the port calls in a non-decreasing sequence. This makes high quality routings for smaller instances of the LSNDP, but for large scale instances it becomes necessary to select a small cluster of related ports in order to efficiently solve the integer program used in the heuristic. A different matheuristic approach is seen in Brouer et al. (2014b), where the core component in a large scale neighborhood search is an integer program designed to capture the complex interaction of the cargo allocation between routes. The solution of the integer program provides a set of moves in the composition of port calls and fleet deployment. Metaheuristics for the LSNDP are challenged by the difficulty of predicting the changes in the multi-commodity flow problem for a given move in the solution space without reevaluating the MCF at the lower tier. The approach of Brouer et al. (2014b) relies on estimation functions of changes in the flow and the fleet deployment related to inserting or removing a port call from a given service and network configuration. Flow changes and the resulting change in the revenue are estimated by solving a series of shortest path problems on the residual graph of the current network for relevant commodities to the insertion/removal of a port call along with an estimation of the change in the vessel related cost with the current fleet deployment.

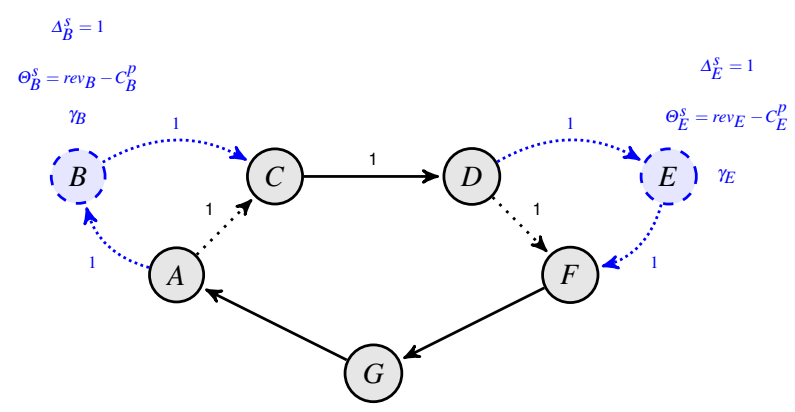

(a) Nodes $B, E$ (blue/dashed) are evaluated for insertion corresponding to variables $\gamma_{i}$ for the set of ports in the neighborhood $N^{s}$ of service $s$

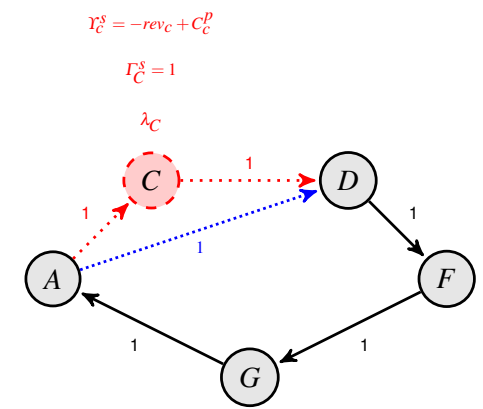

(b) Node $C$ (red/dashed) is evaluated for removal corresponding to variables $\lambda_{i}$ for the set of current port calls $F^{s}$ on service $s$.

Fig. 4 Illustration of the estimation functions for insertion and removal of port calls.

Given a total estimated change in revenue of $r e v_{i}$ and port call cost of $C_{i}^{p}$ Figure 4(a) illustrates estimation functions for the change in revenue $\left(\Theta_{i}^{S}\right)$ and duration $\left(\Delta_{i}^{S}\right)$ increase for inserting port $i$ into service 
$s$ controlled by the binary variable $\gamma_{i}$. The duration controls the number of vessels needed to maintain a weekly frequency of service. Figure 4(b) illustrate the estimation functions for the change in revenue $\left(\Upsilon_{i}^{s}\right)$ and decrease in duration $\left(\Gamma_{i}^{s}\right)$ for removing port $i$ from service $s$ controlled by the binary variable $\lambda_{i}$. Insertions/removals will affect the duration of the service in question and hence the needed fleet deployment modeled by the integer variable $\omega_{s}$ representing the change in the number of vessels deployed. The integer program (9)-(16) expresses the neighborhood of a single service, $s$.

$$
\begin{array}{ll}
\max & \sum_{i \in N^{s}} \Theta_{i}^{s} \gamma_{i}+\sum_{i \in F^{s}} \Upsilon_{i}^{s} \lambda_{i}-C_{V}^{e(s)} \omega_{s} \\
\text { s.t. } & T_{s}+\sum_{i \in N^{s}} \Delta_{i}^{s} \gamma_{i}-\sum_{i \in F^{s}} \Gamma_{i}^{s} \lambda_{i} \leq 24 \cdot 7 \cdot\left(n_{s}^{e(s)}+\omega_{s}\right) \\
& \omega_{s} \leq M_{e(s)} \\
& \sum_{i \in N^{s}} \gamma_{i} \leq I_{s} \\
& \sum_{i \in F^{s}} \lambda_{i} \leq R_{s} \\
& \sum_{j \in L_{i}} \lambda_{j} \leq\left|L_{i}\right|\left(1-\gamma_{i}\right) \quad i \in N^{s} \\
& \sum_{j \in L_{i}} \lambda_{j} \leq\left|L_{i}\right|\left(1-\lambda_{i}\right) \quad i \in F^{s} \\
& \lambda_{i} \in\{0,1\}, i \in F^{s}, \quad \gamma_{i} \in\{0,1\}, i \in N^{s}, \quad \omega_{s} \in \mathbb{Z} .
\end{array}
$$

The objective function (9) accounts for the expected change in revenue of the considered insertions and removals along with the weekly vessel $\operatorname{cost} C_{V}^{e(s)}$ of the vessel class $e(s)$ deployed to service $s$. Constraint (10) considers the expected change in the duration of the service, where $T_{s}$ is the current duration and $n_{s}^{e(s)}$ is the number of vessels currently deployed to service $s$. The possible addition of vessels is bounded by the number of vessels available $M_{e(s)}$ of type $e$ in constraint (11). A limit on the number of insertions/removals respectively are introduced in constraints (12)-(13) to reduce the error of the estimation functions for multiple insertions/removals. The estimation functions additionally depend on the existing port calls for unloading the commodities introduced by the insertions as well as the ports used for rerouting commodities when removing ports. This is handled by introducing a lockset $L_{i}$ for each insertion/removal expressed in constraints (14)-(15). The integer program is solved iteratively for each service in the current network, and the resulting set of moves is evaluated for acceptance in a simulated annealing framework. The procedure is an improvement heuristic (Archetti and Speranza, 2014) fine tuning a given network configuration. The algorithm in its entirety constructs an initial network using a simple greedy construction heuristic. The improvement heuristic is applied as a move operator for intensification of the constructed solution. To diversify the solution a perturbation step is performed at every tenth loop through the entire set of services. The perturbation step alters the service composition in the network by removing entire services with low utilization and introducing a set of new services based on the greedy construction heuristic for undeployed vessels. To evaluate the matheuristic the public benchmark suite, LINER-LIB, for liner shipping network design problems is used.

\section{Speed Optimization}

A key decision related to the operation of a shipping network is the sailing speed between the serviced ports. As fuel costs may constitute more than $75 \%$ of the total operating cost of a vessel (Ronen, 2011) the sailing 
speeds across the network has a significant impact on the operating costs of a network. Hence, it is also one of the planning problems where level of service considerations will impact the configuration substantially. Higher sailing speeds will yield shorter transit times for the customers, but will at the same time result in a more expensive network operation illustrating the inherent trade-off in operating a low cost network versus a network which is optimized in terms of cargo transit times. Overall changes in sailing speed will also affect the strategic decisions regarding the required fleet size in the longer perspective.

Bunker consumption for a vessel profile is often modeled as a cubic function of speed, but in practice it depends not only on the speed of operation, but also on the vessel type, the draft of the vessel (e.g., the actual load), the number of reefer containers powered by the vessel's engine, and weather conditions. During a round trip the vessel may sail at different speeds between ports. The vessel may slow steam to save bunker fuel or increase speed to meet a crucial transit time. Speed may be constrained not only by the vessel design, but also by hard weather conditions or navigation through difficult areas.

In the literature, Notteboom and Vernimmen (2009) provide detailed background information on the influence of bunker cost on liner shipping network configurations and describe how carriers changed sailing speed and number of vessels deployed when bunker fuel cost increased. Ronen (2011) and Corbett et al. (2009) also conclude that the optimal sailing speed is likely to be reduced when the fuel price is increased. In addition to this, Ronen (2011) presents a model to determine the best average sailing speed and number of vessels for a specific sequence of port calls. Wang and Meng (2012) consider a liner shipping network and present a non-linear MIP model to optimize the speed of the deployed vessels. However, container routing is only considered on a set of pre-defined container routes where all demand must be met, and no level of service requirement is considered. Kim (2013) present a model for a liner shipping service to minimize bunker costs and inventory costs for a single vessel. The model determines the bunkering ports as well as speed and number of vessels deployed on the service. No level of service requirements are imposed leading to very slow sailing speeds. There is a lack of methods for optimizing sailing speed on individual sailing legs in a network while considering level of service requirements and maximization profit. This is also the case for methods considering sailing optimization as part of a fleet deployment model. Gelareh and Meng (2010) develop a model to allocate vessels to a set of predefined routes such that they minimize the fleet operating costs but they do not consider level of service requirements. They determine the frequencies and the sailing speeds necessary to meet the demand. Meng and Wang (2011b) also allocate vessels and find sailing speeds for each leg in a single service while minimizing the daily operating costs, but again, no level of service requirements are imposed.

In the context of tramp shipping, Wen et al. (2015) solve the simultaneous optimization of routing and sailing speed using a branch-and-price algorithm with heuristic column generation. The real-life test instances are solved to (near) optimality. Fagerholt et al. (2010) optimize the speed on each leg of a single fixed route where the sequence of ports and the time windows for each visited port are fixed. Norstad et al. (2011) and Gatica and Miranda (2011) solve the simultaneous speed optimization and routing problem for the less-than-shipload and full-shipload problems in tramp shipping. The routing and scheduling problem in tramp shipping is similar to the vehicle routing problem (Laporte et al., 2013). Routing and scheduling in liner shipping has very different characteristics due to the natural multi-commodity flow structure of the liner shipping network and only some of the ideas from tramp shipping can be adapted.

Reinhardt et al. (2016a) present a simple but effective model for speed optimization of an existing liner shipping network. The model adjusts berthing times to smooth out the speed of the vessels assuming that routes and sequence of port calls are fixed. The objective is to minimize fuel consumption while retaining the customer transit times including the transshipment times. To avoid too many changes to the time table, a penalty is associated with each change. Bunker consumption is assumed to grow cubically with the speed, but to ensure a linear model the bunker consumption is approximated by a number of linear secants.

Let $Q$ be the set of demands which are to be transported by the shipping company. Each demand $q \in Q$ contains an origin, destination, amount of containers, duration of transport and a price. The duration for the transport of the demand from origin to destination is in the remainder of this survey referred to as 
transit time. The company has a set of routings used for shipping the demand. These routings should not be changed to ensure that capacity constraints, cabotage rules and embargoes are satisfied as before.

When rescheduling the port visits the transit time of a demand may change. It is important that the company can make sure that the transit time stays within their requirements so that customers are not lost. The transit time of a demand $q \in Q$ is denoted as $\bar{T}_{q}$.

The model assumes a weekly operation, so the schedule is repeated every $24 \cdot 7=168$ hours. A penalty is paid for each change in port calls to ensure that only the most advantageous port calls are changed.

Let $R$ be the set of services, and $L$ be the set of legs where $l \in L_{r}$ is a leg on service $r \in R$. Let $Q$ be the set of demands defined by a route (a set of legs $l \in L$ ) between two ports $X$ and $Y$ and $C$ the set of connections between service $l \in L$ and $\hat{l} \in L$ used by the demands in $Q$. As the routes for each demand in $Q$ are known in terms of the legs used on each service and also the connections between services, two additional sets are used to account for the transit time of a route for a demand; the set $\left(l, l^{\prime}\right) \in L_{q}$, where we define $l$ to be the port call preceeding $l^{\prime}$, and $C_{q}$ to be the set of connections used by demand $q$. finally, let $S_{l}$ be the set of secants used for approximating the bunker curve on leg $l \in L$.

Define $B_{l}$ as the penalty for shifting a berth time at leg $l . W_{r}$ is the number of weeks used for the round trip of a service. $P_{l}^{\text {stay }}$ is the port stay of leg $l \in L . P_{l}^{\text {pilot }}$ is the time used for piloting on leg $l \in L . D_{l}$ is the distance of leg $l \in L . T_{l}$ is the current scheduled time for the berth visit. $T_{l}^{\text {min }}$ is the minimum time used for sailing leg $l \in L$ (at maximum speed). $\hat{T}_{c}^{\text {min }}$ is the minimum time required for connection $c \in C$. $\hat{T}_{c}^{\text {weeks }}$ is equivalent to $\hat{T}_{c}^{\text {min }} \bmod 168$ for connection $c \in C$. The consumption of bunker $\phi_{l}^{p}$ is the gradient of secant $p \in P$ of leg $l \in L . \omega_{l}^{p}$ is the $y$-axis intersection of secant $p \in P$ of leg $l \in L$.

Finally, let $f_{l} \in\{0,1\}$ be a binary variable indicating if leg $l$ is a start leg of a service. Let $t_{l}^{d} \geq 0$ express the departure time of leg $l \in L$ at its end port and $t_{l}^{a} \geq 0$ express the arrival time of leg $l \in L$ at its end port. $o_{l} \geq 0$ is the fuel cost of sailing leg $l \in L . w_{l} \in \mathbb{Z}$ denotes the number of weeks from start of leg $l \in L$. This means that $w_{l}=t_{l} \bmod 168 . a_{c} \in\{0,1\}$ is one iff $w_{l}>t_{l}^{a}-\hat{T}_{c}^{\min } \bmod 168$ for $c=(h, l) \in C . m_{l} \in\{0,1\}$ is one iff the port visit time has been changed. $x_{c} \in \mathbb{Z}$ indicates the number of weeks needed to make the connection.

$$
\min \sum_{r \in R} \sum_{l \in L_{r}} o_{l}+B_{l} m_{l}
$$

$$
\begin{array}{lr}
\text { s.t. } & r \in R \\
\sum_{l \in L_{r}} f_{l}=1, & r \in R,\left(l^{\prime}, l\right) \in L_{r} \\
t_{l}^{a}-t_{l^{\prime}}^{d}-P_{l}^{\text {pilot }}+168 W_{r} f_{l} \geq T_{l}^{\text {min }}, & r \in R, l \in L_{r} \\
T_{l}-\left(t_{l}^{d}-168\left(w_{l}\right)+168 m_{l}\right) \geq 0, & r \in R, l \in L_{r} \\
t_{l}^{d}-168 w_{l}-T_{l}+168 m_{l} \geq 0, & p \in P, r \in R,\left(l^{\prime}, l\right) \in L_{r}, s \in S_{l} \\
\phi_{l}^{p}\left(t_{l}^{a}-t_{l^{\prime}}^{d}-P_{l}^{\text {pilot }}+168 W_{r} f_{l}\right)+\omega_{l}^{p} \leq o_{l}, & r \in R, l \in L_{r} \\
0 \leq t_{l}^{d}-168 w_{l}<168, & c=(h, l) \in C \\
t_{l}^{a}-168\left(w_{l}-a_{c}\right)-\hat{T}_{c}^{\text {min }} \geq 0, & c=(l, h) \in C \\
t_{h}^{a}-t_{l}^{d}-\hat{T}_{c}^{\text {min }}-168\left(w_{h}-w_{l}-x_{c}-a_{c}\right) \geq 0, & \\
\quad \sum_{c=(l, h) \in C_{q}}\left(t_{h}^{a}-t_{l}^{d}-168\left(w_{h}-w_{l}-x_{c}-\hat{T}_{c}^{\text {weeks }}\right)\right. & r \in R, q \in Q \\
\quad+\sum_{\left(l, l^{\prime}\right) \in L_{q}}\left(t_{l}^{d}-t_{l^{\prime}}^{d}+168 W_{r} f_{l}\right)-P_{q}^{\text {stay }} \leq \bar{T}_{q}, & r \in R, l \in L_{r}
\end{array}
$$




$$
\begin{aligned}
& t_{l}^{a}, t_{l}^{d} \geq 0, t_{l}^{d} \geq 0, o_{l} \geq 0, \\
& f_{l}, m_{l}, a_{c} \in\{0,1\} \\
& x_{c} \in\{0,1,2\}
\end{aligned}
$$

$$
\begin{array}{r}
r \in R, l \in L_{r} \\
c \in C, r \in R, l \in L_{r} \\
c \in C
\end{array}
$$

The objective function (17) minimizes the sum of the fuel cost and penalties for moving port call times. The parameter $B$ is the penalty for moving the port call time at the end of leg $l \in L$, and the binary variable $m_{l}$ is 1 iff the port call time has been moved. The parameter $C_{l}$ is the bunker cost on leg $l$.

Constraints (18) ensure that exactly one leg is chosen as the first for each service. Constraints (19) ensure that the leg speed does not exceed the maximum speed of the vessel. Constraints (20) and (21) invokes a penalty if the port time is changed from the original scheduled (weekly) port visit time $T_{l}$ for leg $l \in L$. For every leg, the consumption is restricted by a set of linear functions represented by constraints (22). In constraints (22) the variable $\phi_{l}^{p}$ is the slope of secant $p_{l}$ on leg $l \in L$ and $\omega_{l}^{p}$ is the intersection of the secant. Constraints (23) define the value of $w_{l}$. Constraints (24) ensure that transit times are correctly accounted for when $t_{l}^{a}-\hat{T}_{c}^{\text {min }}$ is in the previous week and thus sets $a_{c}$ if required. Constraints (25) ensure that the container waits at the terminal for the next vessel arriving after the minimum required connection time $\hat{T}_{c}^{\text {min }}$ and sets the variable $x_{c}$ accordingly. The transit time is ensured to be below the requirement $\bar{T}_{q}$ for demand $q \in Q$ with constraint (26). Constraints (27) to (30) define the variable domains. The variables (28) are continuous variables indicating the time and cost.

Reinhardt et al. (2016a) report that the model is able to save around $2 \%$ of bunker consumption while keeping all transit times unchanged. If transit times can be extended by up to 48 hours, a saving of around $6-7 \%$ is achievable. The CPU time is in both cases limited to 10 minutes.

Karsten et al. (2015b) present a speed optimization model, where the container routings are not fixed and may change as a consequence of the speed changes on each leg. The network as such is fixed, but the speed on each leg is allowed to vary and hence commodities may take different paths if speed changes allow for a cheaper or faster route than currently available. The problem is solved using Benders' decomposition and results indicate that the flow changes significantly along with the individual leg speeds, however, execution times are much higher than solving the simple speed optimization problem presented in Reinhardt et al. (2016a). Karsten et al. (2015a) incorporate variable speed on each leg into the network design process, by extending the matheuristic from Karsten et al. (2016) with variable speed on the services. The leg speeds are iteratively calculated for each single service taking the current flow of containers into account. The method adjusts speed to the required transit times of the current container routings throughout the roundtrip and takes into account speed changes in order to meet transit time restrictions of rejected demand. The individual leg speeds are calculated using a MIP with the objective of minimizing a piece wise linear approximation to the cubic bunker consumption function.

\section{Empty container repositioning}

In extension of the network design process, a liner shipping company must also consider revenue management at a more operational level. Requests for cargo can be rejected if it is not profitable to carry the containers, or if bottlenecks in the network make it infeasible. Moreover, empty containers tend to accumulate at importing regions due to a significant imbalance in world trade. Therefore, repositioning empty containers to exporting regions imposes a large cost on liner shippers, and these costs need to be incorporated in the revenue model. In Song and Dong (2014) it is stated that the global cost of repositioning empty containers in 2009 was more than 20 billion USD and constituted more than $20 \%$ of global port handling activities. Since larger shipping companies at any time have several millions of containers in circulation, balancing the empty containers from import to export regions is an extremely complex task and requires advanced solution methods. Epstein et al. (2012) describe a complex decision support system for reposi- 
tioning and stocking empty containers at a large liner shipping company (CSAV), resulting in savings of $\$ 81$ million on their fleet of 700.000 TEU containers by increasing container turnover by $60 \%$ and reducing inventory stock by $50 \%$. Erera et al. (2005) study the empty repositioning of a fleet of tank containers in a time-extended uncapacitated network. They advocate the simultaneous optimization of routing current bookings and the derived empty repositioning to achieve higher utilization of tank containers and minimize cost for the operator. Meng and Wang (2011a) study a network design problem selecting among a set of candidate shipping lines while considering the container routing problem along with the repositioning of empty containers. The model presented in Erera et al. (2005) handles loaded end empty containers simultaneously, however load rejection is not allowed and the aim is to minimize the cost of transporting all empty and laden containers. Song and Dong (2012) consider a problem of joint cargo routing and empty container repositioning at the operational level accounting for the demurrage and inventory cost of empty containers. Like most other works on empty repositioning it is a cost minimizing problem where load rejection is not allowed and in order to limit the complexity of the cargo routing problem laden containers are only allowed two transshipments.

Brouer et al. (2011) present a revenue management model for strategic planning within a liner shipping company. A mathematical model is presented for maximizing the profit of cargo transportation, while considering the possible cost of repositioning empty containers. The model allows load rejection effectively considering whether the repositioning of an empty container is profitable.

The booking decision of a liner shipper considering empty container repositioning can be described as a specialized multi-commodity flow problem with inter-balancing constraints to control the flow of empty containers.

Similarly to the pure cargo routing problem we can define a commodity as the tuple $\left(O_{k}, D_{k}, q_{k}, r_{k}\right)$ representing a demand of $q_{k}$ in number of containers from node $O_{k}$ to node $D_{k}$ with a sales price per unit of $r_{k}$. The unit cost of arc $(i, j)$ for commodity $k$ is denoted $c_{i j}^{k}$. The non-negative integer variable $x_{i j}^{k}$ is the flow of commodity $k$ on arc $(i, j)$. The capacity of $\operatorname{arc}(i, j)$ is $u_{i j}$. To model the empty containers an empty super commodity $k_{e}$ is introduced. The flow of the empty super commodity is defined for all $(i, j) \in A$ as the integer variables $x_{i j}^{k_{e}}$. The unit cost of arc $(i, j)$ for commodity $k_{e}$ is denoted $c_{i j}^{k_{e}}$. The empty super commodity has no flow conservation constraints and appear in the objective with a cost and in the bundled capacity and inter-balancing constraints. For convenience the commodity set is split into the loaded commodities and the empty super commodity: Let $K_{F}$ be the set of loaded commodities. Let $K_{e}$ be the set of the single empty super commodity. Finally, let $K=K_{F} \cup K_{e}$. The inter-balancing constraints additionally introduce a new set of variables representing leased containers at a node. The cost of leasing is modeled in the objective. Let $c_{l}^{i}$ be the cost of leasing a container at port $i$, while $l_{i}$ is the integer leasing variable at port $i$. Demand may be rejected, due to capacity constraints and unprofitability from empty repositioning cost. The slack variable $\gamma_{k}$ represents the amount of rejected demand for commodity $k$.

\subsection{Path Flow Formulation}

In the following we introduce a path flow model which is an extension of model (5)-(8). The model is extended by an empty container flow defined for all arcs, inter-balancing constraints at every node to ensure the repositioning of empty containers to points of interest. The model also contains the ability to lease containers in nodes at a cost. Again, let $p$ be a path connecting $O_{k}$ and $D_{k}$, and $P_{k}$ be the set of all paths belonging to commodity $k$. The flow on path $p$ is denoted by the variable $f^{p}$. The binary coefficient $a_{i j}^{p}$ is 1 if and only if arc $(i, j)$ is on the path $p$. Finally, $c_{p}^{k}=\sum_{(i, j) \in A} a_{i j}^{p} c_{i j}^{k}$ is the cost of path $p$ for commodity $k$. The variable $l_{i}$ models the number of leased containers at node $i$ and $c_{l}^{i}$ is the cost of leasing a container in node $i$. The master problem is: 


$$
\begin{aligned}
& \max \sum_{k \in K_{F}} \sum_{p \in P_{k}}\left(r_{k}-c_{p}^{k}\right) f^{p}-\sum_{(i, j) \in A} c_{i j}^{k_{e}} x_{i j}^{k_{e}}-\sum_{i \in N} c_{l}^{i} l^{i} \\
& \text { s.t. } \sum_{k \in K_{F}} \sum_{p \in P_{k}} a_{i j}^{p} f^{p}+x_{i j}^{k_{e}} \leq u_{i j} \quad(i, j) \in A \\
& \sum_{p \in P_{k}} f^{p}+\gamma_{k}=q_{k} \quad k \in K_{F} \\
& \sum_{k \in K_{F}} \sum_{p \in P_{k}} \sum_{j \in N}\left(a_{i j}^{p}-a_{j i}^{p}\right) f^{p}+\sum_{j \in N}\left(x_{i j}^{k_{e}}-x_{j i}^{k_{e}}\right)-l^{i} \leq 0 \quad i \in N \\
& f^{p} \in \mathbb{Z}_{+}, p \in P_{k}, \quad \gamma_{k} \in \mathbb{Z}_{+}, k \in K_{F} \quad x_{i j}^{k_{e}} \in \mathbb{Z}_{+},(i, j) \in A, \quad l^{i} \in \mathbb{Z}_{+}, i \in N
\end{aligned}
$$

where the $x_{i j}^{k}$ variables can be replaced by $\sum_{p \in P_{k}} a_{i j}^{p} f^{p}$ for all $k \in K_{F}$. Constraints (34) balance the number of leased, empty and full containers at every node and basically determines the flow of empty containers and leased containers needed to ensure a container for every full container transported. The convexity constraints for the individual subproblems (33) bound the flow between the $\left(O_{k}, D_{k}\right)$ pair from above (a maximal flow of $q_{k}$ is possible).

Paths are generated on the fly using delayed column generation. Brouer et al. (2011) report computational results for eight instances based on real life shipping networks, showing that the delayed column generation algorithm for the path flow model clearly outperforms solving the arc flow model with the CPLEX barrier solver. In order to fairly compare the arc and path flow formulation a basic column generation algorithm is used for the path flow model versus a standard solver for the arc flow model. Instances with up to 234 ports and 293 vessels for 9 periods were solved in less than 35 minutes with the column generation algorithm. The largest instance solved for 12 periods contains 151 ports and 222 vessels and was solved in less than 75 minutes.

The algorithm solves instances with up to 16,000 commodities over a twelve month planning period within one hour. Integer solutions are found by simply rounding the LP solution. The model of Erera et al. (2005) is solved to integer optimality using standard solvers as opposed to the rounded integer solution presented here. The problem sizes of Brouer et al. (2011) are significantly larger than those of Erera et al. (2005) and the rounded integer solutions lead to a gap of at most $0.01 \%$ from the LP upper bound of the path flow formulation, which is very acceptable, and far below the level of uncertainty in the data. The results of Erera et al. (2005) confirm the economic rationale in simultaneously considering loaded and empty containers. Song and Dong (2014) provides an in-depth review of the literature on empty container repositioning problems for the interested reader.

\section{Stowage planning}

With vessels carrying up to 20,000 TEU, stowage of the containers on board is a non-trivial task demanding fast algorithms as the final load list is known very late. Stowage planning can be split into a master planning problem and a more detailed slot planning problem. The master planning problem should decide a proper mixture of containers, so that constraints on volume, weight, and reefer plugs are respected. The slot planning problem should assign containers to slots in the vessel so that the loading and unloading time in ports can be minimized. The vessel must be seaworthy, meaning that stability and stress constraints must be respected.

Figure 5 illustrates the arrangement of bays in a container vessel. Containers are loaded bottom-up in each bay up to a given stacking height limited by the line of sight and other factors. Some containers are loaded below deck, while other containers are loaded above the hatch cover. The overall weight sum of 


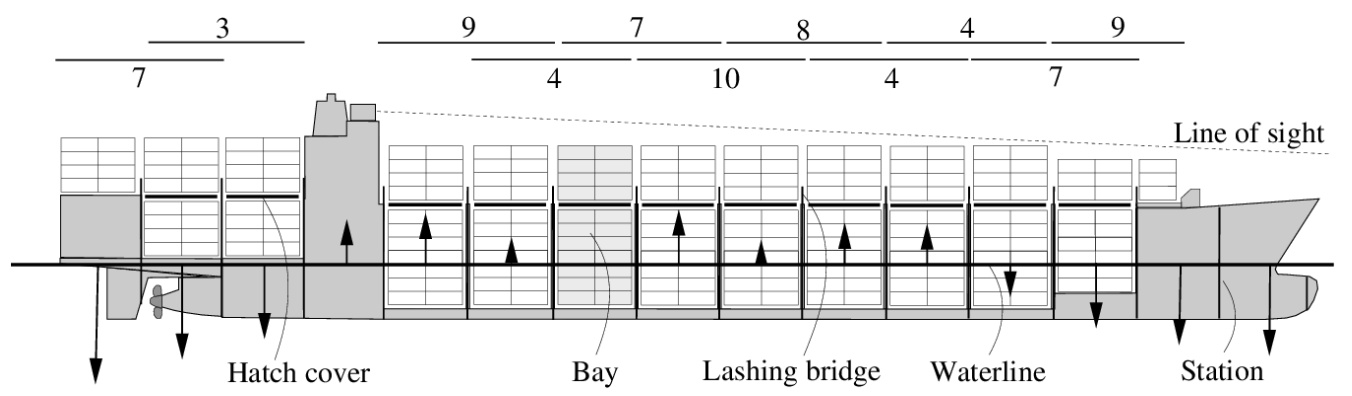

Fig. 5 The arrangement of bays in a small container vessel, and stacking heights. The arrows indicate forces. Picture: Pacino (2012).

containers may not exceed a given limit, and the weight needs to be balanced. Moreover, stress forces on the vessel should be limited, making it illegal to, e.g., only load containers at the same front and end of the vessel. Refrigerated containers (reefers) need to be attached to an electric plug. Only a limited number of plugs are available, and these plugs are at specific positions.

A good stowage plan should make sure that it is not necessary to rearrange containers at each port call. All containers for the given port should be directly accessible when arriving to the port, and there should be sufficient free capacity for loading new containers. If several cranes are available in a port, it is necessary to ensure that all cranes can operate at the same time without blocking for each other and that they have roughly the same make-span of operation.

Pacino (2012) presents a MIP model for the master problem. The model is based on Pacino et al. (2011); Pacino and Jensen (2012). The model considers both 20' and 40' containers, assuming that two 20' containers can fit in the slot of a 40' container provided that the middle is properly supported. Four types of containers are considered: light, heavy, light reefer, and heavy reefer. Decision variables are introduced for each bay, indicating how many of each container type will be loaded in the bay.

The MIP model has a large number of constraints: First of all, a load list and cargo estimates are used to calculate the number of containers of each type that needs to be stowed. Moreover, every bay has a capacity of dry containers and reefers. An overall weight limit given by the capacity of the vessel is also imposed. When calculating the weight limit, average values for light and heavy containers are used to ease the calculations.

Trim, draft, buoyancy and stability is calculated as a function of displacement and center of gravity of the vessel.

Finally, a number of penalties associated with a given loading are calculated. These include hatchoverstowage, overstowage in slots, time needed for loading, and excess of reefer containers. The objective of the model minimizes a weighted sum of the penalties.

Pacino (2012) shows that the master planning problem is NP-hard. Computational results are reported for instances with vessel capacity up to around 10,000 TEU, visiting up to 12 ports involving more than 25,000 lifts (crane moves of a container). Several of these instances can be solved within 5 minutes up to a $5 \%$ gap, using a MIP-solver.

\subsection{Mathematical model}

In the slot planning phase, the master plan is refined by assigning the containers to specific slots in a bay on board the vessel (Pacino and Jensen, 2013). This problem involves handling of a number of stacking rules, as well as constraints on stack heights and stack weight. Since several of the containers are already stowed 
on board the vessel, the objective is to arrange containers with the same destination port in the same stack, free as many stacks as possible, minimize overstowage, and minimize the number of non-reefer containers assigned to reefer slots. Due to the large number of logical constraints in this problem Delgado et al. (2012) proposed a logical model using the following notation. $S$ is the set of stacks, $T_{S}$ is the set of tiers for stack $s, P$ represents the aft $(p=1)$ and fore $(p=2)$ of a cell, $C$ is the set of containers to stow in the location and $C^{P} \subset C$ is the subset of containers in the release, i.e., the set of containers that are already on-board the vessel.

Let $x_{s t p c}$ be a binary variable indicating whether container $c$ is placed at location $s \in S, t \in T_{s}, p \in P$. Moreover, we use the auxilary variable $h_{s t}^{\prime} \geq 0$ to denote the max height of the two containers at position $p=1$ and $p=2$.

For each location, given by stack $s$, tier $t$, and position $p$, we have a number of binary parameters: $A_{s t p}^{40}$ is one if the location can hold a 40' container, and similarly $A_{s t p}^{20}$ is one if the location can hold a 20 ' container. Furthermore, $A_{s t p}^{R}$ is one iff the position is equipped with a power plug for a reefer container.

For each container $c$, we have three binary parameters: $r_{c}$ is one if the container is a reefer container, $f_{c}$ is one if the container is a 40' container, and $t_{c}$ is one if it is a 20 ' container. The continuous parameters $w_{c}$ and $h_{c}$ denote the weight and height of the container. Finally, we have a parameter $v_{c}$ denoting the profitability of the container.

For every stack $s$ we have the parameters $W_{s}$ and $H_{s}$ denoting the maximum weight and height. This leads to the following MIP model, adapted from the logical model in Pacino (2012):

$$
\begin{aligned}
& \max \sum_{s \in S} \sum_{t \in T_{s}} \sum_{p \in P} \sum_{c \in C} v_{c} x_{s t p c} \\
& \text { s.t. } \sum_{s \in S} \sum_{t \in T_{s}} \sum_{p \in P} x_{s t p c} \leq 1 \quad c \in C \\
& \sum_{c \in C} x_{s t p c} \leq 1 \quad s \in S, t \in T_{s}, p \in P \\
& t_{c} x_{s t p c} \leq A_{s t p}^{20} \quad c \in C, s \in S, t \in T_{s}, p \in P \\
& f_{c} x_{s t 1 c} \leq A_{s t 1}^{40} \quad c \in C, s \in S, t \in T_{s} \\
& \sum_{c \in C} x_{s t 2 c} \leq 1-\sum_{c \in C} f_{c} x_{s t 1 c} \quad s \in S, t \in T_{s} \\
& \sum_{t \in T_{s}} \sum_{c \in C}\left(w_{c} x_{s t 1 c}+w_{c} x_{s t 2 c}\right) \leq W_{s} \quad s \in S \\
& \sum_{c \in C} h_{c} x_{s t 1 c} \leq h_{s t}^{\prime} \quad s \in S, t \in T_{s} \\
& \sum_{c \in C} h_{c} x_{s t 2 c} \leq h_{s t}^{\prime} \quad s \in S, t \in T_{s} \\
& \sum_{t \in T_{s}} h_{s t}^{\prime} \leq H_{s} \quad s \in S \\
& \sum_{c \in C} t_{c} x_{s(t+1) 1 c} \leq 1-\sum_{c \in C} f_{c} x_{s t 1 c} \quad s \in S, t \in T_{s} \backslash\left\{N_{s}^{T}\right\}, \\
& \sum_{c \in C} t_{c} x_{s(t+1) 2 c} \leq 1-\sum_{c \in C} f_{c} x_{s t 1 c} \quad s \in S, t \in T_{s} \backslash\left\{N_{s}^{T}\right\}, \\
& \sum_{c \in C} r_{c} t_{c} x_{s t p c} \leq A_{s t p}^{R} \quad s \in S, t \in T_{s}, p \in P \\
& \sum_{c \in C} r_{c} f_{c} x_{s t p c} \leq A_{s t 1}^{R}+A_{s t 2}^{R} \quad s \in S, t \in T_{s}, p \in P
\end{aligned}
$$




$$
x_{s t p c} \in\{0,1\}, h_{s t p}^{\prime} \geq 0 \quad s \in S, t \in T_{s}, p \in P, c \in C
$$

Constraints (37) ensure that each container is assigned to at most one location, and constraints (38) ensure that each location can hold at most one container.

The next constraints (39) and (40) make sure that a 20' container only can be assigned to a position which can hold a 20' container, and similarly for a 40' container. Moreover, constraints (41) say that if a 40 ' container is assigned to position 1, then no container can be assigned to position 2 at the given location. Constraints (42) make sure that the weight constraint of each stack is not exceeded, and constraints (45) ensure the same for the height. The help constraints (43) and (44) calculate $h^{\prime}$ as the maximum height of the two containers in position 1 and 2. Constraints (46) and (47) forbid that two 20' containers are stacked on top of a 40' container. Finally, constraints (48) and (49) make sure that reefer containers are assigned to slots with a power plug.

Delgado et al. (2012) use a constraint formulation of the above model in combination with ConstraintBased Local Search in order to minimize the objective function. The framework combines local search algorithms with constraint programming. The constraint satisfaction part of the problem is transformed to an optimization problem where the objective is to minimize constraint violation. A hill-climbing method is used to optimize the slot planning. The neighborhood in the search consists of swapping containers between a pair of cells.

Pacino (2012) reports computational results for 133 real-life instances, showing that the local search algorithm actually finds the optimal solution in $86 \%$ of the cases. The running times are below 1 second.

\section{Bunker purchasing}

According to Wang and Teo (2013) bunker fuel costs account up to 50-60\% of a ship's total operating cost in times of high fuel prices, and the inventory holding costs of the bunker on board add further expenses to the liner shipping company. Optimal strategies for bunker purchasing are therefore essential for efficient operation of a liner shipping network.

Bunker prices are fluctuating and generally correlated with the crude oil price, but there are significant price differences between ports. This creates the need for frequent (daily) re-optimization of the bunker plan for a vessel, to ensure the lowest bunker costs.

Bunker can be purchased on the spot market when arriving to a port, but normally it is purchased some weeks ahead of arrival. Long-term contracts between a liner shipping company and a port can result in reduced bunkering costs by committing the company to purchase a given amount of bunker. Bunkering contracts may cover several vessels sailing on different services, making the planning quite complex.

The bunker purchasing problem is to satisfy the vessels consumption by purchasing bunkers at the minimum overall cost, while considering reserve requirements, and other operational constraints. Real-life instances of the bunker purchasing problem may involve more than 500 vessels, 40,000 port calls, and 750 contracts.

For a vessel sailing on a given port to port voyage at a given speed, the bunker consumption can be fairly accurately predicted. This gives an advantage in bunker purchasing, when a vessel has a stable schedule known for some months ahead. The regularity in the vessel schedules in liner shipping allows for detailed planning of a single vessel.

Besbes and Savin (2009) consider different re-fueling policies for liner vessels and present some interesting considerations on the modelling of stochastic bunker prices using Markov processes. This is used to show that the bunkering problem in liner shipping can be seen as a stochastic capacitated inventory management problem. Capacity is the only considered operational constraint. 
The work of Plum and Jensen (2007) considers multiple tanks in the vessel and stochasticity of both prices and consumption, as well as a range of operational constraints. Yao et al. (2012) does not consider stochastic elements nor tanks, but vessel speed is variable. The work of Kim et al. (2012) minimizes bunker costs as well as startup costs and inventory costs for a single liner shipping vessel. This is done by choosing bunker ports and bunker volumes but also having vessel round trip speed (and thus the number of vessels on the service) as a variable in the model.

In Sheng et al. (2014), a model is developed which considers the uncertainty of bunker prices and bunker consumption, modelling the uncertainty by Markov processes in a scenario tree. The work can be seen as an extension of (Yao et al., 2012), as it considers vessel speed as a variable within the same time window bounds. Capacity and fixed bunkering costs are considered, as is the holding / tied capital cost of the bunkers.

Finally, Wang and Teo (2013) study re-planning of network configuration and financial hedging of bunker fuel prices. These risk management measures are the two most important measures employed by container shipping companies to reduce bunker fuel price risk.

\subsection{Contracts}

Plum et al. (2015) presented a decomposition algorithm for the Bunker Purchasing with Contracts Problem (BPCP), and showed that the model is able to solve even very large real-life instances. The model is based on writing up all bunkering patterns, and hence may be of exponential size. Let $I$ be the set of ports visited on an itinerary, $B$ be the set of bunker types, and $V$ be the set of vessels. A contract $c \in C$ has a minimal $\underline{u}_{c}$ and maximal $\bar{u}_{c}$ quantity that needs to be purchased. A contract $c$ will give rise to a number of purchase options $m \in M$, i.e., discrete events where a specific vessel $v$ calls a port within the time interval of a contract $c$, allowing it to purchase bunker at the specific price $p_{m}$. Each time a purchase is done at port $i$ a startup $\operatorname{cost} S_{i}$ is paid.

Let $R_{v}$ be the set of all feasible bunkering patterns for a vessel $v$. A bunkering pattern is feasible if a sufficient amount of bunker is available for each itinerary, including reserves. Bunker is available in various grades, and it is allowed to substitute a lower grade with a higher grade. In some areas, only low-sulphur bunker may be used, and this needs to be respected by the bunkering plan. Moreover initial and terminal criteria for bunker volumes must be met. Finding a legal bunkering pattern can be formulated as a MIP model (Plum et al., 2015) and solved by commercial solvers. Each pattern $r \in R_{v}$ is denoted as a set of bunkerings.

Let $h_{r}=\sum_{m \in M}\left(p_{m} l_{m}\right)+\sum_{i \in I} \sum_{b \in B}\left(S_{i} \delta_{i, b}\right)$ be the cost for pattern $r \in R_{v}$. In this expression, $l_{m}$ is the purchase of bunker for each purchase option $m$. and $p_{m}$ is the price of option $m$. The binary variable $\delta_{i, b}$ is set to one iff a purchase of bunker type $b$ is made at port call $i$. Let $\lambda_{r}$ be a binary variable, set to one iff the bunkering pattern $r$ is used. Let $o_{r, c}$ be the purchased quantity of contract $c$ by pattern $r$. The BPCP can then be formulated as

$$
\begin{aligned}
& \min \sum_{v \in V} \sum_{r \in R_{v}} h_{r} \lambda_{r}+\sum_{c \in C}\left(\underline{w} \underline{s}_{c}+\bar{w} \bar{s}_{c}\right) \\
& \text { s.t. } \quad \underline{u}_{c}-\underline{s}_{c} \leq \sum_{v \in V} \sum_{r \in R_{v}} o_{r, c} \lambda_{r} \leq \bar{u}_{c}+\bar{s}_{c} \quad c \in C \\
& \sum_{r \in R_{v}} \lambda_{r}=1 \quad v \in V \\
& \lambda_{r} \in\{0,1\} \quad r \in R_{v}
\end{aligned}
$$


The objective minimizes the costs of purchased bunker, startup costs and slack costs. The parameters $\underline{w}$ and $\bar{w}$ denote a penalty for violating the minimal $\underline{u}_{c}$ and maximal $\bar{u}_{c}$ quantity imposed by contract $c$. Constraints (52) ensures that all contracts are fulfilled. Convexity constraints (53) ensure that exactly one bunker pattern is chosen for each vessel.

Due to the large number of columns in the model, Plum et al. (2015) proposed to solve the LP relaxed model by Column Generation. Using the generated columns from the LP-solution, the resulting problem is solved to integer optimality using a MIP solver, leading to a heuristic solution for the original problem.

Initially all dual variables are set to zero, a subproblem is constructed for each vessel and solved as a MIP problem. The first master problem is then constructed with one solution for each vessel as columns. This master is solved and the first values are found. The subproblems are resolved for all vessels (only the objective coefficients for the contracts needs updating) and new columns are generated for the master. This continues until no negative reduced cost columns can be generated, and the LP optimal solution is achieved.

The subproblems need not be solved to optimality since any column with negative reduced cost will ensure progress of the algorithm. Therefore the solver is allowed to return solutions to the subproblem having a considerable optimality gaps. As the algorithm progresses, the allowable subproblem gap is reduced.

A simple form of dual stabilization has been used in the implementation by (Plum et al., 2015) to speed up convergence. The Box-step method imposes a box around the dual variables, which are limited from changing more than $\pi_{\max }$ per iteration. This has been motivated by the dual variables only taking on values $\{-\underline{w}, \bar{w}, 0\}$ in the first iteration, these then stabilize at smaller numerical values in subsequent iterations.

The model is able to solve even very large real-life instances involving more than 500 vessels, 40,000 port calls, and 750 contracts. First, column generation is used to solve the linearized model, and then a MIP solver is used to find an integer solution only using the generated columns. This results in a small gap in the optimal solution compared to the case where all columns were known. However, computational results show that the gap is never more than around $0.5 \%$ even for the largest instances. In practice the resulting gap of the algorithm, can be much smaller since the found solutions are benchmarked against a lower bound and not against the optimal solution.

An interesting side product of the model is the dual variables $\underline{\pi}_{c}$ and $\bar{\pi}_{c}$ for the upper and lower contract constraints (52). These values can be used to evaluate the gain of a given contract, which may be valuable information when (re)negotiating contracts.

Since bunker prices are of stochastic nature, future research should be focused on modeling the price fluctuation. However, the models tend to become quite complex and difficult to solve as observed by Plum and Jensen (2007), while only adding small extra improvements to the results. So a trade-off must be done between model complexity and gain in bunker costs. The work of Sheng et al. (2014) shows some promising developments in this important direction.

Also, instruments from finance (bunker future or forward contracts, fixed price bunker fuel swaps) could be used to control risk in bunker purchasing, and to increase the margins on oil trade. Bunker purchasing for liner ships constitutes such a big market that it deserves a professional trading approach.

\section{The vessel schedule recovery problem}

It is estimated that approximately $70-80 \%$ of vessel round trips experience delays in at least one port. The common causes are bad weather, strikes in ports, congestions in passageways and ports, and mechanical failures.

Currently the operator at the shipping companies manually decides what action to take in case of a disruption. For a single delayed vessel a simple approach could be to speed up. However, the consumption of bunker fuel is close to a cubic function of speed, and vessels' speeds are limited between a lower and upper limit. So even though an expensive speed increase strategy is chosen, a vessel can arrive late for 
connections, propagating delays to other parts of the network. Having more than 10,000 containers on board a large vessel, the overall consequences of re-routing/delaying these containers is too complex for a human and a quantitative decision support tool can aid in identifying re-routing options and cost. Disruption management is well studied within the airline industry (see Ball et al. (2007) or Clausen et al. (2010) for a review) and the network design of airlines resemble liner shipping networks inspiring the few works on disruption management found for liner shipping. Mulder et al. (2012) presents a Markov decision model to determine the optimal recovery policy. The core idea is to reallocate buffer time within a schedule in order to recover from disruptions. Brouer et al. (2013) present the Vessel Schedule Recovery Problem (VSRP) handling a disruption in a liner shipping network by omitting port calls, swapping port calls or speeding up vessels in a predefined disruption scenario. The model and method of this paper will be presented in the following section.

\subsection{Mathematical formulation}

Let $V$ be the set of vessels and $P$ the set of ports in a disruption scenario delimited by a recovery time horizon $T$. The terminal cargo handling crews operate in shifts which are paid for in full, even if arriving in the middle of a shift. Hence we only allow vessels arriving at the beginning of shifts. The recovery time horizon reflects these shifts by defining discrete time slots $t \in T$ corresponding to the beginning of a shift. Reducing the graph to time-slots based on these shifts also has the advantage of reducing the graph size, although this is a minor simplification of the problem. For each vessel $v \in V$, the current location and a planned schedule consisting of an ordered set of port calls $H_{v} \subseteq P$ are known within the recovery horizon, a port call $A$ can precede a port call $B, A<B$ in $H_{v}$. A set of possible sailings, i.e., directed edges, $L_{h}$ are said to cover a port call $h \in H_{v}$. Each $L_{h}$ represents a sailing with a different speed.

The recovery horizon, $T$, is an input to the model given by the user, based on the disruption in question. Intercontinental services will often recover by speeding during ocean crossing, making the arrival at first port after an ocean crossing a good horizon, severe disruptions might require two ocean crossings. Feeders recovering at arrival to their hub port call would save many missed transshipments giving an obvious horizon. In combination with a limited geographical dimension this ensures that the disruption does not spread to the entire network.

The disruption scenario includes a set of container groups $C$ with planned transportation scenarios on the schedules of $V$. A feasible solution to an instance of the VSRP is to find a sailing for each $v \in V$ starting at the current position of $v$ and ending on the planned schedule no later than the time of the recovery horizon. The solution must respect the minimum and maximum speed of the vessel and the constraints defined regarding ports allowed for omission or port call swaps. The optimal solution is the feasible solution of minimum cost, when considering the cost of sailing in terms of bunker and port fees along with a strategic penalty on container groups not delivered "on-time" or misconnecting altogether.

\subsection{Time-Space Network}

Brouer et al. (2013) use a time space graph, modelling the network of ports $P$ in the disruption scenario. The edges of the graph model the set of available recovery techniques, which are applicable to the VSRP, such as port call swaps and port call omissions.

Let $G=(N, E)$ be a directed graph with node set $N=\left\{p^{t} \in N \mid p \in P, t \in T\right\}$ where $p^{t}$ denotes port $p$ at time $t$. Let $n^{-}$and $n^{+}$denote the in- and out-going edges of node $n \in N$, respectively. Let $N_{v} \subseteq N$ be the set of all nodes for vessel $v \in V$. The set consists of a source node $n_{s}^{v}$ corresponding to the current position 
of the vessel and a sink node $n_{t}^{v}$ corresponding to the scheduled position at the end of the recovery horizon. Additional nodes are created for the set of port calls $h \in H_{v}$ within a time window of $\left\{a_{v}^{h}, b_{v}^{h}\right\}$ defining the earliest and latest arrival time respectively given the vessels minimum and maximum speed, the current position and the remaining set of port calls.

The binary variables $x_{e}$ for each edge $e \in E_{s}$ are set to one iff the edge is sailed in the solution. Binary variables $z_{h}$ for each port call $h \in H_{v} \quad v \in V$ are set to one iff call $h$ is omitted. For each container group $c$ we define binary variables $o_{c}$ to indicate whether the container group is delayed or not, and $y_{c}$ to account for container groups misconnecting. $B_{c} \in H_{v}$ is defined as the origin port for a container group $c \in C$ and the port call where vessel $v$ picks up the container group. Similarly, we define $T_{c} \in H_{w}$ as the destination port for container group $c \in C$ and the port call where vessel $w$ delivers the container group. The binary parameter $O_{e}^{c}$ is one iff container group $c \in C$ is delayed when arriving by edge $e \in L_{T_{c}}$. Intermediate planned transshipment points for each container group $c \in C$ are defined by the ordered set $I_{c}=\left(I_{c}^{1}, \ldots, I_{c}^{m}\right)$. Here $I_{c}^{i}=\left(h_{v}^{i}, h_{w}^{i}\right) \in\left(H_{v}, H_{w}\right)$ is a pair of calls for different vessels $(v, w \in V \mid v \neq w)$ constituting a transshipment. Each container group $c$ has $m^{c}$ transshipments. $M_{c}^{e}$ is the set of all non-connecting edges of $e \in L_{h}$ that result in miss-connection of container group $c \in C . M_{c} \in \mathbb{Z}_{+}$is an upper bound on the number of transshipments for container group $c \in C$.

Let the demand of vessels $v$ in a node $n$ be given by $S_{v}^{n}=-1$ if $n=n_{s}^{v}, S_{v}^{n}=1$ if $n=n_{t}^{v}$, while $S_{v}^{n}=0$ for all other nodes. Then we get the following model:

$$
\begin{aligned}
& \min \sum_{v \in V} \sum_{h \in H_{v}} \sum_{e \in L_{h}} c_{e}^{v} x_{e}+\sum_{c \in C}\left(c_{c}^{m} y_{c}+c_{c}^{d} o_{c}\right) \\
& \text { s.t. } \sum_{e \in L_{h}} x_{e}+z_{h}=1 \quad v \in V, h \in H_{v} \\
& \sum_{e \in n^{-}} x_{e}-\sum_{e \in n^{+}} x_{e}=S_{v}^{n} \quad v \in V, n \in N_{v} \\
& y_{c} \leq o_{c} \quad c \in C \\
& \sum_{e \in L_{T_{c}}} O_{e}^{c} x_{e} \leq o_{c} \quad c \in C \\
& z_{h} \leq y_{c} \quad c \in C, h \in B_{c} \cup I_{c} \cup T_{c} \\
& x_{e}+\sum_{\lambda \in M_{c}^{e}} x_{\lambda} \leq 1+y_{c} \quad c \in C, e \in\left\{L_{h} \mid h \in B_{c} \cup I_{c} \cup T_{c}\right\} \\
& x_{e} \in\{0,1\}, e \in E_{s} \quad y_{c}, o_{c} \in \mathbb{R}_{+}, c \in C \quad z_{h} \in \mathbb{R}_{+}, v \in V, h \in H_{v}
\end{aligned}
$$

The objective function (55) minimizes the cost of operating vessels at the given speeds, the port calls performed along with the penalties incurred from delaying or misconnecting cargo.

Constraints (56) are set-partitioning constraints ensuring that each scheduled port call for each vessel is either called by some sailing or omitted. The next constraints (57) are flow-conservation constraints. Combined with the binary domain of variables $x_{e}$ and $z_{h}$ they define feasible vessel flows through the time-space network. A misconnection is by definition also a delay of a container group and hence the misconnection penalty is added to the delay penalty, as formulated in (58). Constraints (59) ensure that $o_{c}$ takes the value one iff container group $c$ is delayed when arriving via the sailing represented by edge $e \in E_{s}$. Constraints (60) ensure that if a port call is omitted, which had a planned (un)load of container group $c \in C$, the container group is misconnected. Constraints (61) are coherence constraints ensuring the detection of container groups' miss-connections due to late arrivals in transshipment ports. On the left-hand side the decision variable corresponding to a given sailing, $x_{e}$, is added to the sum of all decision variables corresponding to having onward sailing resulting in miss-connections, $\lambda \in M_{c}^{e}$. 
In (Brouer et al., 2013) the model has been tested on a number of real-life cases, including a delayed vessel, a port closure, a berth prioritization, and expected congestion. An analysis of the four real life cases show that a disruption allowing to omit a port call or swap port calls may ensure timely delivery of cargo without having to increase speed. Hence, a decision support tool based on the VSRP may aid in decreasing the number of delays in a liner shipping network, while maintaining a slow steaming policy. To operationalize this, the rerouting of the actual flow and adjustment of the actual schedule must be incorporated in a real time system to enable here-and-now decisions. This is especially challenging for larger disruption scenarios than the ones described as the size of the problem grows exponentially.

\section{Computational results using LINER-LIB}

LINER-LIB 2012 is a public benchmark suite for the LSNDP presented by Brouer et al. (2014a). The data instances of the benchmark suite are constructed from real-life data from the largest global linershipping company, Maersk Line, along with several industry and public stakeholders. LINER-LIB consists of seven benchmark instances available at http://www.linerlib.org (see Brouer et al. (2014a) for details on the construction of the data instances). Each instance can be used in a low, medium, and high capacity case depending on the fleet of the instance. Table 1 presents some statistics on each instance ranging from smaller networks suitable for optimal methods to large scale instances spanning the globe. Currently published results are available for 6 of the 7 instances, leaving the WorldLarge instance unsolved.

\begin{tabular}{|c|c|c|c|c|c|c|}
\hline \multirow{3}{*}{$\begin{array}{l}\text { Category } \\
\text { Single-hub instances }\end{array}$} & Instance and description & $|\mathbf{P}|$ & $\mathbf{K} \mid$ & $|E|$ & \multicolumn{2}{|c|}{$\min v \max v$} \\
\hline & Baltic Baltic sea, Bremerhaven as hub & 12 & 22 & 2 & 5 & 7 \\
\hline & WAF West Africa, Algeciras as hub & 19 & 38 & 2 & 33 & 51 \\
\hline Multi-hub instance & $\begin{array}{l}\text { Mediterranean Mediterranean, Algeciras, Tangier, Gioia } \\
\text { Tauro as hubs }\end{array}$ & 39 & 369 & 3 & 15 & 25 \\
\hline Trade-lane instances & Pacific (Asia-US West) & 45 & 722 & 4 & 81 & 119 \\
\hline & AsiaEurope Europe, Middle East, and Far east regions & 111 & 4000 & 6 & 140 & 212 \\
\hline World instance & $\begin{array}{l}\text { Small } 47 \text { Main ports worldwide identified by Maersk Line } \\
\text { Large } 197 \text { ports worldwide identified by Maersk Line }\end{array}$ & $\begin{array}{r}47 \\
197\end{array}$ & $\begin{array}{l}1764 \\
9630\end{array}$ & $\begin{array}{l}6 \\
6\end{array}$ & $\begin{array}{l}209 \\
401\end{array}$ & $\begin{array}{l}317 \\
601\end{array}$ \\
\hline
\end{tabular}

Table 1 The instances of the benchmark suite with indication of the number of ports $(|P|)$, the number of origin-destination pairs $(|K|)$, the number of vessel classes $(|E|)$, the minimum $(\min v)$ and maximum number of vessels $(\max v)$.

LINER-LIB contains data on ports including port call cost, cargo handling cost and draft restrictions, distances between ports considering draft and canal traversal, vessel related data for capacity, cost, speed interval and bunker consumptions, and finally a commodity set with quantities, revenue, and maximal transit time. The commodity data reflects the current imbalance of world trade and the associated differentiated revenue. It is tailored for models of the LSNDP, but may provide useful data for related maritime transportation problems.

Computational results for LINER-LIB are presented in Plum et al. (2014); Brouer et al. (2014a,b). Brouer et al. (2014a) presented the first results for the benchmark suite using the reference model (Brouer et al., 2014a) with biweekly frequencies for the feeder vessel classes and weekly frequencies for remaining classes. The heuristic column generation algorithm is used to solve all instances but the WorldLarge instance with promising results. Brouer et al. (2014b) present computational results using the reference model with weekly frequencies for all vessel classes which has a more restricted solution space than Brouer et al. (2014a). As a consequence the solutions from Brouer et al. (2014b) are feasible for the model used in Brouer et al. (2014a), but not vice-versa. However, the computational results of Brouer et al. (2014b) indicate that the matheuristic using an improvement heuristic based on integer programming scales well 
for large instances and holds the current best known results for the Pacific, WorldSmall and AsiaEurope instances. Plum et al. (2014) present a service flow model for the LSNDP using a commercial MIP solver presenting results for the two Baltic and WAF instances of LINER-LIB. For details on the results the reader is referred to the respective papers. LINER-LIB is currently used by researchers at a handful of different universities worldwide and may provide data for future results on models and algorithms for LSNDP.

\subsection{Updated results}

LINER-LIB is being used by several peer researchers and their work has pointed to a few implementation errors in the matheuristic. This section contains corrected and updated results according to their findings. There are currently three known sources of errors in the results from Brouer et al. (2014b):

- Parsing demands above 1000 TEU in the WorldSmall instance has been interpreted differently in Brouer et al. (2014a) than in Brouer et al. (2014b) as a thousand seperator has been interpreted as a comma in Brouer et al. (2014b) resulting in a lower total demand volume.

- When scaling the weekly objective value to the planning horizon of 180 days an error results in only scaling by 175 days ( 25 weeks) instead of 180 days as in Brouer et al. (2014a) due to integer division of 180 instead of division in $\mathbb{R}$.

- In the original results the construction heuristic allows services with a longer port stay than 24 hours in order to maintain the weekly frequency requirement. It means that the vessel is allowed more idle time at port and unfortunately, the fuel cost during this idling time has not been accounted for.

In the paper Brouer et al. (2014b) new best results where found for ten instances. We have fixed the above mentioned issues in the code. For the instances of the matheuristic having the current best known results and where one or more services in the best found network did include extra idling time, new tests have been run with the errors corrected. For the remaining instances with one or more services having extra idle time the cost of extra idling in ports over the planning horizon was added to the objective value if relevant. Likewise, we have made runs with corrected input for WorldSmall containing the demand quantity used in WorldSmall of Brouer et al. (2014a) - this instance is denoted WorldSmall*. Table 2 reports the corrected results for all instances and show that the performance of the algorithm is not greatly influenced by the errors. Results actually improve for most instances except AsiaEurope.

We are gratefull for our peer researchers drawing our attention to issues and errors, and apologize for any inconvenience caused. All best known networks along with an erratasheet can be found on the LINERLIB git hub repository, which is available to the public at https://github.com/BBrouer/Linerlib.

\section{Conclusion}

We have given a detailed overview of optimization problems in liner shipping, covering both modeling aspects, methodology, and applications. Large-scale mathematical models have been presented, addressing operational (cargo routing), tactical (speed optimization and service selection), and strategic (network design) planning problems faced by liner shipping companies.

The global container transportation network has grown to a size where humans alone cannot easily assess the best configuration and hence they must rely on advanced decision support tools in the decision process to attain the most competitive network. Using the advanced planning tools presented in this survey, a liner shipping company will quickly be able to assess the impact of their decisions and get an overview of the ripple effects across the network. 


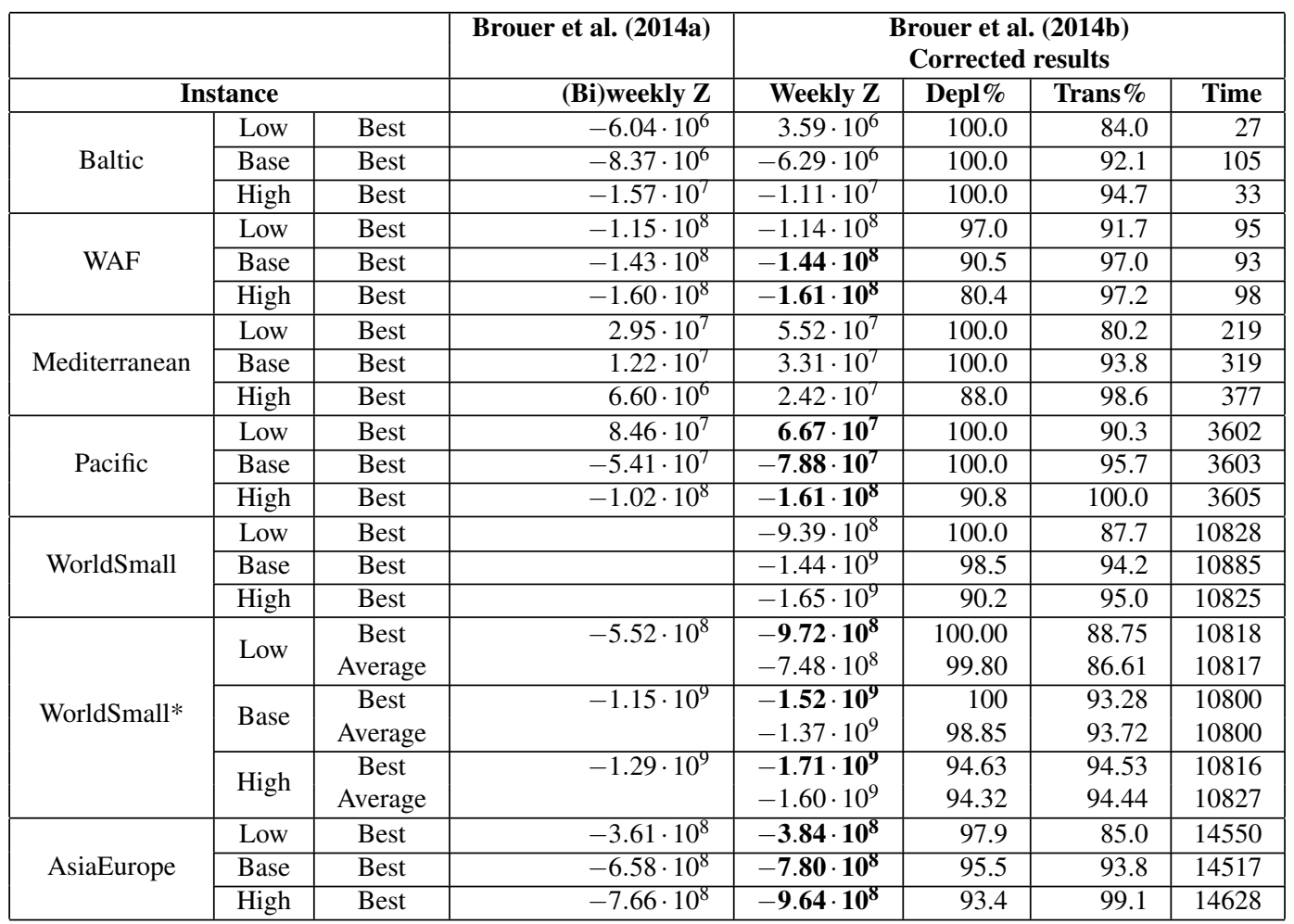

Table 2 Overview of the updated results obtained for the benchmark suite LINER-LIB 2012 using the matheuristic with all known errors corrected and a new WorldSmall instance, denoted WorldSmall*, where demand quantity is equal to that of Brouer et al. (2014a). The tests are based on ten runs with different random seeds. THe objective is to minimize operational costs. Instance is the name of the instance including Low, Base and High to indicate the low-, meidum, and high-capacitated cases; Best refers to the best solution found; Average is the average over all ten runs; Brouer et al. (2014a) specifies the objective value obtained in Brouer et al. (2014a) allowing biweekly frequencies for vessel classes below 1200 FFE - for WorldSmall demand quantities are not comparable and the comparison is omitted; Weekly $\mathbf{Z}$ is the objective value obtained by the proposed matheuristic allowing weekly frequencies for all vessel classes; Depl\% is the percentage of the total number of available vessels deployed; Trans\% is the percentage of demand transported; Time is the CPU time in seconds. Numbers in bold highlight the solutions with a lower (i.e. better) objective value than in Brouer et al. (2014a).

From a practical perspective having tools that support incremental changes and improvements is of high value as they can help managers and planners to quickly adapt to new markets, changing demands or fuel costs, as well as when negotiating cooperation agreements.

According to Notteboom (2006) around 2,7\% of total CO2 emission stem from liner shipping, so every improvement in the various parts of supply chain are important to maintain liner shipping as a competitive and green mode of transportation.

\subsection{Improved solution methods}

There are several ways to extend or even improve the methods presented in this survey. From a planner perspective this could yield faster methods making the decision process more agile. From a shipper perspective better solutions will make the carriers able to offer more competitive products at the same or even lower price. Related to the cargo routing problem, it is relevant to consider methods that can help speed up the 
solution as the problem is evaluated often. The "tailing off effect" is limited when solving the cargo flowing multi-commodity flow problem discussed in Section 2.1, but a stabilization scheme on the dual variables may still improve the convergence (Lübbecke and Desrosiers, 2005). Furthermore, it would be interesting to test alternative solution methods for the multi-commodity flow problem. Babonneau et al. (2006) propose a method with good performance when bottlenecks appear in a network, which is the case for several sailing legs in a global network. The method is based on a Lagrangian relaxation restricted to the arcs that are likely to be saturated at the optimum. These will be relatively easy to identify in a liner shipping network, e.g., canals and head haul on main trades and could be a competitive alternative to the proposed method in terms of computational time, but it may be more complicated to consider level of service requirements.

\subsection{Future challenges}

Sulphur emission control areas, SECA, or emission control areas, ECA, are areas in the sea where stricter controls are established to minimize airborne emissions such as SOx (sulphur oxides), NOx (nitrogen oxides), ODS (ozone depleting substances), and VOC (volatile organic compounds) from ships. The impact of these on the network design varies from region to region, but one way to handle them in the design process is to have two alternative available routes/edges in the graph (as in Fagerholt and Psaraftis (2015)) in relevant areas.

Along the same lines, cabotage rules apply to commercial ships in most countries and are implemented to protect the domestic shipping industry from foreign competition, hence these are quite important to consider both during the design process and especially when evaluating flows.

In the current literature only transit times are considered. However, the path flow formulation of the multi-commodity flow problem can easily be extended to also explicitly limit the number of transshipments by including a resource that keeps track of the number of transshipment edges used.

A more detailed cargo mix (reefers, high cube, 20', 40', etc.) can also be handled in the cargo routing problem by including additional capacity constraints in the multi-commodity flow problem and a specification of the container type in the demand list.

The more detailed the level of service aspects can be considered in the decision support tools the more tailored products can be offered to customers and it is possible to differentiate customers according to level of service. Consequently it will be possible for a liner shipping company to request higher transportation costs from customers and possibly even attract new customers.

The report by Joerss et al. (2015) points out that container lines successfully have used alliances to increase scope and reduce cost on the ocean. Hence cooperation in landside operation is the next logical step for capturing economies of scale. Reinhardt et al. (2016b) is a first attempt in this direction, but further models and decision support tools are needed that can support this development.

Finally, it is important to remember that the shipping part is only a part of the global supply chain, and all links can introduce delays and uncertainty. Therefore, decision support tools should, both from a carrier and customer perspective, ideally integrate uncertainty and robustness considerations and manage a larger part of the supply chain to be able to eventually offer better and more robust products to both producers and consumers. This will require additional extensions of the application, modeling, and methodology. 


\section{Acknowledgments}

The authors would like to thank the Danish Strategic Research Council for having supported the projects ENERPLAN and GREENSHIP, and the Danish Maritime Fund for having supported the project Competitive Liner Shipping Network Design.

\section{References}

R. Agarwal and Ö. Ergun. Ship scheduling and network design for cargo routing in liner shipping. Transportation Science, 42(2):175-196, 2008.

J. F. Alvarez. Joint routing and deployment of a fleet of container vessels. Maritime Economics \& Logistics, 11:186-208, 2009.

C. Archetti and M. Grazia Speranza. A survey on matheuristics for routing problems. EURO Journal on Computational Optimization, 2:223-246, 2014.

F.c Babonneau, O. Du Merle, and J-P Vial. Solving large-scale linear multicommodity flow problems with an active set strategy and proximal-accpm. Operations Research, 54(1):184-197, 2006.

A. J. Baird. Optimising the container transhipment hub location in northern europe. Journal of Transport Teography, 14(3):195-214, 2006.

A. Balakrishnan and C. V. Karsten. Joint service selection and cargo routing with limited transshipments. Submitted to: Annals of Operations Research: Logistics, Optimization, and Transportation, 2015a.

A. Balakrishnan and C. V. Karsten. Optimal selection of liner containership services with limited transshipments. Submitted to: European Journal of Operations Research, 2015b.

M. Ball, C. Barnhart, G. Nemhauser, and A. Odoni. Handbooks in Operations Research \& Management Science: Transportation, volume 14, chapter 1: Air Transportation - Irregular Operations and Control. Elseview, 2007.

C. Barnhart, E. L. Johnson, G. L. Nemhauser, M. WP. Savelsbergh, and P. H. Vance. Branch-and-price: Column generation for solving huge integer programs. Operations research, 46(3):316-329, 1998.

O. Besbes and S. Savin. Going bunkers: The joint route selection and refueling problem. Manufacturing and Service Operations Management, 11:694-711, 2009.

R.E. Bixby. A brief history of linear and mixed-integer programming computation. Documenta Mathematica, Optimization Stories:107-121, 2012.

B. D. Brouer, C. V. Karsten, and D. Pisinger. Big data optimization in maritime logistics. In Ali Emrouznejad, editor, Big Data Optimization: Recent Developments and Challenges, volume 18 of Studies in Big Data, pages 319-344. Springer, 2016.

B.D. Brouer, D. Pisinger, and S. Spoorendonk. Liner shipping cargo allocation with repositioning of empty containers. INFOR: Information Systems and Operational Research, 49:109-124, 2011.

B.D. Brouer, J. Dirksen, D. Pisinger, C.E.M. Plum, and B. Vaaben. The vessel schedule recovery problem (VSRP) a MIP model for handling disruptions in liner shipping. European Journal of Operational Research, 224:362-374, 2013.

Berit D Brouer, J Fernando Álvarez, Christian EM Plum, David Pisinger, and Mikkel M Sigurd. A base integer programming model and benchmark suite for liner-shipping network design. Transportation Science, 48(2):281-312, 2014a.

Berit Dangaard Brouer, Guy Desaulniers, and David Pisinger. A matheuristic for the liner shipping network design problem. Transportation Research Part E: Logistics and Transportation Review, 72:42-59, $2014 \mathrm{~b}$.

E.K. Burke and G. Kendall. Search Methodologies: Introductory Tutorials in Optimization and Decision Support Techniques. Springer Science, 2014. 
Marielle Christiansen, Kjetil Fagerholt, Bjørn Nygreen, and David Ronen. Ship routing and scheduling in the new millennium. European Journal of Operational Research, 228(3):467-483, 2013.

J. Clausen, A. Larsen, J. Larsen, and N. J. Rezanova. Disruption management in the airline industryconcepts, models and methods. Computers and Operations Research, 37(5):809-821, 2010.

James J Corbett, Haifeng Wang, and James J Winebrake. The effectiveness and costs of speed reductions on emissions from international shipping. Transportation Research Part D: Transport and Environment, 14(8):593-598, 2009.

A. M. Costa. A survey on benders decomposition applied to fixed-charge network design problems. Computers \& Operations Research, 32(6):1429-1450, 2005.

A. Delgado, R. M. Jensen, K. Janstrup, T. H. Rose, and K. H. Andersen. A constraint programming model for fast optimal stowage of container vessel bays. European Journal of Operational Research, 220(1): 251-261, 2012.

J. Desrosiers and M. E. Lübbecke. A primer in column generation. Springer, 2005.

R. Epstein, A. Neely, and A. Weintraub. A strategic empty container logistics optimization in a major shipping company. Interfaces, 42(1):5-16, 2012.

A. L. Erera, J.C. Morales, and M. Savelsbergh. Global intermodal tank container management for the chemical industry. Transportation Research Part E, 41(6):551-566, 2005.

Kjetil Fagerholt. Optimal fleet design in a ship routing problem. International Transactions in Operational Research, 6(5):453-464, 1999.

Kjetil Fagerholt, Gilbert Laporte, and Inge Norstad. Reducing fuel emissions by optimizing speed on shipping routes. Journal of the Operational Research Society, 61(3):523-529, 2010.

K.1 Fagerholt and H. N. Psaraftis. On two speed optimization problems for ships that sail in and out of emission control areas. Transportation Research Part D: Transport and Environment, 39:56-64, 2015.

Ricardo A Gatica and Pablo A Miranda. Special issue on latin-american research: A time based discretization approach for ship routing and scheduling with variable speed. Networks and Spatial Economics, 11 (3):465-485, 2011.

Shahin Gelareh and Qiang Meng. A novel modeling approach for the fleet deployment problem within a short-term planning horizon. Transportation Research Part E: Logistics and Transportation Review, 46 (1):76-89, 2010.

Shahin Gelareh, Stefan Nickel, and David Pisinger. Liner shipping hub network design in a competitive environment. Transportation Research Part E: Logistics and Transportation Review, 46(6):991-1004, 2010.

M. Gendreau and J-Y Potvin. Handbook of metaheuristics, volume 2. Springer, 2010.

M. Joerss, J. Murnane, S. Saxon, and R. Widdows. Landside operations: The next frontier for containershipping alliances. Technical report, McKinsey \& Company, 2015.

C. V. Karsten, B. D. Brouer, and D. Pisinger. Competitive liner shipping network design. Submitted to: Computers and Operations Research, 2015a.

C. V. Karsten, D. Pisinger, and S. Ropke. Simultaneous optimization of liner shipping vessel speed and container routing with transit time restrictions. Submitted to: Transportation Science, 2015b.

C. V. Karsten, D. Pisinger, S. Ropke, and B. D. Brouer. The time constrained multi-commodity network flow problem and its application to liner shipping network design. Transportation Research Part E: Logistics and Transportation Review, 76:122-138, 2015c.

C. V. Karsten, D. Pisinger, B. D. Brouer, and G. Desaulniers. Time constrained liner shipping network design. Transportation Research Part E: Coordination and Control in Transport Logistics, 2016. doi: 10.1016/j.tre.2016.03.010.

H-J Kim, Y-T Chang, K-T Kim, and H-J Kim. An epsilon-optimal algorithm considering greenhouse gas emissions for the management of a ships bunker fuel. Transportation Research Part D: Transport and Environment, 17:97 - 103, 2012. 
HJ Kim. A lagrangian heuristic for determining the speed and bunkering port of a ship. Journal of the Operational Research Society, 65(5):747-754, 2013.

Karina H Kjeldsen. Classification of ship routing and scheduling problems in liner shipping. INFOR: Information Systems and Operational Research, 49(2):139-152, 2011.

Gilbert Laporte, Paolo Toth, and Daniele Vigo. Vehicle routing: historical perspective and recent contributions. EURO Journal on Transportation and Logistics, 2(1-2):1-4, 2013.

Marco E Lübbecke and Jacques Desrosiers. Selected topics in column generation. Operations Research, 53(6):1007-1023, 2005.

Q. Meng and S. Wang. Liner shipping service network design with empty container repositioning. Transportation Research Part E: Logistics and Transportation Review, 47(5):695-708, $92011 \mathrm{a}$.

Qiang Meng and Shuaian Wang. Optimal operating strategy for a long-haul liner service route. European Journal of Operational Research, 215(1):105-114, $2011 \mathrm{~b}$.

Qiang Meng, Shuaian Wang, Henrik Andersson, and Kristian Thun. Containership routing and scheduling in liner shipping: overview and future research directions. Transportation Science, 48(2):265-280, 2014.

J. Mulder, R. Dekker, and M. Sharifyazdi. Designing robust liner shipping schedules: Optimizing recovery actions and buffer times. Report / Econometric Institute, Erasmus University Rotterdam, 2012. URL http://hdl.handle.net/1765/38636.

Inge Norstad, Kjetil Fagerholt, and Gilbert Laporte. Tramp ship routing and scheduling with speed optimization. Transportation Research Part C: Emerging Technologies, 19(5):853-865, 2011.

T.E. Notteboom. The time factor in liner shipping services. Maritime Economics \& Logistics, 8(1):19-39, 2006.

Theo Notteboom and Jean-Paul Rodrigue. Containerisation, box logistics and global supply chains: The integration of ports and liner shipping networks. Maritime Economics \& Logistics, 10(1):152-174, 2008.

Theo E Notteboom and Bert Vernimmen. The effect of high fuel costs on liner service configuration in container shipping. Journal of Transport Geography, 17(5):325-337, 2009.

D Pacino. Fast generation of container vessel stowage plans. Ph. D. Thesis, IT University of Copenhagen, 2012.

D. Pacino and R.M. Jensen. Constraint-based local search for container stowage slot planning. Lecture Notes in Engineering and Computer Science, 2:1467-1472, 2012.

D. Pacino and R.M. Jensen. Fast slot planning using constraint-based local search. IAENG Transactions on Engineering Technologies, 186:49-63, 2013.

D. Pacino, A. Delgado, R.M. Jensen, and T. Bebbington. Fast generation of near-optimal plans for ecoefficient stowage of large container vessels. Computational Logistics, 6971:286-301, 2011.

D. Pisinger. Liner shipping network design - a new decomposition. In 28th European Conference on Operational Research, 2016.

C.E.M. Plum, D. Pisinger, and M. M. Sigurd. A service flow model for the liner shipping network design problem. European Journal of Operational Research, 235(2):378-386, 2014.

C.E.M. Plum, D. Pisinger, and P.N. Jensen. Bunker purchasing in liner shipping. In Handbook of Ocean Container Transport Logistics, volume XVII, pages 251-278. Springer, 2015.

Christian E. M. Plum and Peter Neergaard Jensen. Minimization of bunker costs. Master thesis, University of Copenhagen, 2007.

Krishan Rana and RG Vickson. Routing container ships using lagrangean relaxation and decomposition. Transportation Science, 25(3):201-214, 1991.

L. B. Reinhardt, C. E.M. Plum, D. Pisinger, M.M. Sigurd, and G. T.P Vial. The liner shipping berth scheduling problem with transit times. Transportation Research Part E: Logistics and Transportation Review, 86:116-128, 2016a.

L.B. Reinhardt, D. Pisinger, S. Spoorendonk, and M.M. Sigurd. Optimization of the drayage problem using exact methods. INFOR: Information Systems and Operational Research, pages 1-19, $2016 \mathrm{~b}$. 
Line Blander Reinhardt and David Pisinger. A branch and cut algorithm for the container shipping network design problem. Flexible Services and Manufacturing Journal, 24(3):349-374, 2012.

David Ronen. The effect of oil price on containership speed and fleet size. Journal of the Operational Research Society, 62(1):211-216, 2011.

F. Rossi, P. Van Beek, and T. Walsh. Handbook of constraint programming. Elsevier, 2006.

$\mathrm{X}$. Sheng, L. Lee, and E. Chew. Dynamic determination of vessel speed and selection of bunkering ports for liner shipping under stochastic environment. OR Spectrum, 36(2):455-480, 2014.

K. Shintani, A. Imai, E. Nishimura, and S. Papadimitriou. The container shipping network design problem with empty container repositioning. Transportation Research Part E: Logistics and Transportation Review, 43(1):39-59, 2007.

D-P Song and J-X Dong. Cargo routing and empty container repositioning in multiple shipping service routes. Transportation Research Part B: Methodological, 46(10):1556-1575, 2012.

D-P Song and J-X Dong. Empty container repositioning. pages 163-208, 2014.

K. Tierney, B. skelsdttir, R. M. Jensen, and D. Pisinger. Solving the liner shipping fleet repositioning problem with cargo flows. Transportation Science, Articles in Advance, 2013. doi: 10.1287/trsc.2013.0515.

J. P. Vielma. Mixed integer linear programming formulation techniques. SIAM Review, 57:3-57, 2015.

Shuaian Wang and Qiang Meng. Sailing speed optimization for container ships in a liner shipping network. Transportation Research Part E: Logistics and Transportation Review, 48(3):701-714, 2012.

X. Wang and C.C. Teo. Integrated hedging and network planning for container shipping's bunker fuel management. Maritime Economics \& Logistics, 15:172-196, 2013.

M Wen, S Ropke, HL Petersen, R Larsen, and OBG Madsen. Full-shipload tramp ship routing and scheduling with variable speeds. Computers \& Operations Research, 2015.

Z. Yao, S. H. Ng, and L. H. Lee. A study on bunker fuel management for the shipping liner services. Computers and Operations Research, 39:1160 - 1172, 2012. 